\title{
Interpretation of MUSIC for Location Detecting of Small Inhomogeneities Surrounded by Random Scatterers
}

\author{
Won-Kwang Park \\ Department of Mathematics, Kookmin University, Seoul 02707, Republic of Korea \\ Correspondence should be addressed to Won-Kwang Park; parkwk@kookmin.ac.kr
}

Received 27 October 2015; Revised 5 January 2016; Accepted 6 January 2016

Academic Editor: Eric Florentin

Copyright (C) 2016 Won-Kwang Park. This is an open access article distributed under the Creative Commons Attribution License, which permits unrestricted use, distribution, and reproduction in any medium, provided the original work is properly cited.

\begin{abstract}
We consider the MUltiple SIgnal Classification (MUSIC) algorithm for identifying the locations of small electromagnetic inhomogeneities surrounded by random scatterers. For this purpose, we rigorously analyze the structure of MUSIC-type imaging function by establishing a relationship with zero-order Bessel function of the first kind. This relationship shows certain properties of the MUSIC algorithm, explains some unexplained phenomena, and provides a method for improvements.
\end{abstract}

\section{Introduction}

One of the purposes of the inverse scattering problem is to identify the characteristics (location, shape, material properties, etc.) of small inhomogeneities from the scattered field or far-field pattern. This problem, which arises in fields such as physics, engineering, and biomedical science, is highly relevant to human life; thus, it remains an important research area. Related works can be found in [1-5] and references therein.

Attempts to address the problem described above have led to the development of the MUltiple SIgnal Classification(MUSIC-) type algorithm to find unknown inhomogeneities and the algorithm has been applied to various problems, for example, detection of small inhomogeneities in homogeneous space [6-9], location identification of small inhomogeneities embedded in a half-space or multilayered medium [10-12], reconstructing perfectly conducting cracks $[13,14]$, imaging of internal corrosion [15], shape recognition of crack-like thin inhomogeneities [16-18] and volumetric extended targets [19-21], and application to the biomedical imaging [22]. We also refer to [23, 24] for a detailed and concise description of MUSIC. Several research efforts have contributed to confirming that MUSIC is a fast and stable algorithm that can easily be extended to multiple inhomogeneities and that does not require specific regularization terms that are highly dependent on the problem at hand. However, its feasibility is only confirmed when the background medium is homogeneous; that is, the imaging performance of MUSIC when unknown inhomogeneities are surrounded by random scatterers remains unknown. In several works [25-28], an inverse scattering problem in random media has been concerned. Specially, mathematical theory of MUSIC for detecting point-like scatterers embedded in an inhomogeneous medium has been concerned in [29]. Motivated by these remarkable works, a more careful investigation of the mathematical theory is still required.

Motivated by the above, MUSIC algorithm has been applied for detecting the locations of small electromagnetic inhomogeneities when they are surrounded by electromagnetic random scatterers and confirmed that it can be applied satisfactorily. However, this only relied on the results of numerical simulations, that is, a heuristic approach to some extent, which is the motivation for the current work. In this contribution, we carefully analyze the mathematical structure of MUSIC-type imaging function and discover some properties. This work is based on the relationship between the singular vectors associated with nonzero singular values of a multistatic response (MSR) matrix and asymptotic expansion formula due to the existence of small inhomogeneities; refer to [23].

This paper is organized as follows. Section 2 introduces the two-dimensional direct scattering problem and an asymptotic expansion formula in the presence of small 
inhomogeneities. In Section 3, MUSIC-type imaging function is introduced. In Section 4, we analyze the mathematical structure of the MUSIC-type imaging function and discuss its properties. In Section 5, we present the results of numerical simulations to support the analyzed structure of MUSIC and Section 6 presents a short conclusion.

\section{Two-Dimensional Direct Scattering Problem}

In this section, we survey a two-dimensional direct scattering problem and introduce an asymptotic expansion formula. For a more detailed description we recommend $[18,23,30]$. Let $\Sigma_{m}, m=1,2, \ldots, M$, be an electromagnetic inhomogeneity with a small diameter $r_{m}$ in two-dimensional space $\mathbb{R}^{2}$. Throughout this paper, we assume that every $\Sigma_{m}$ is expressed as

$$
\Sigma_{m}=\mathbf{z}_{m}+r_{m} \mathbf{B}_{m}
$$

where $\mathbf{z}_{m}$ denotes the location of $\Sigma_{m}$ and $\mathbf{B}_{m}$ is a simple connected smooth domain containing the origin. For the sake of simplicity, we let $\Sigma$ be the collection of $\Sigma_{m}$. Throughout this paper, we assume that inhomogeneities are well separated from each other such that

$$
\omega\left|\mathbf{z}_{m}-\mathbf{z}_{m^{\prime}}\right| \gg 1-\frac{1}{4}=0.75
$$

for all $m, m^{\prime}=1,2, \ldots, M$ and $m \neq m^{\prime}$.

Let us denote $\Delta_{s}, s=1,2, \ldots, S$, as the random scatterer with small radius $r_{s}<r$ and let $\Delta$ be the collection of $\Delta_{s}$. Similarly, we assume that $\Delta_{s}$ is of the form

$$
\Delta_{s}=\mathbf{y}_{s}+r_{s} \mathbf{B}_{m}
$$

As before, suppose that $\Delta_{s} \cap \Delta_{s^{\prime}}=\emptyset$ for all $s, s^{\prime}=1,2, \ldots, S$ and $s \neq s^{\prime}$ and the positions of $\mathbf{y}_{s}$ are random but they are fixed for all frequencies discussed later.

In this work, we assume that every inhomogeneity is characterized by its dielectric permittivity and magnetic permeability at a given positive angular frequency $\omega=2 \pi / \lambda$, where $\lambda$ denotes the wavelength. Let $\varepsilon_{m}, \varepsilon_{s}$, and $\varepsilon_{0}$ be the electric permittivities of $\Sigma_{m}, \Delta_{s}$, and $\mathbb{R}^{2}$, respectively. Then, we can introduce the piecewise-constant electric permittivity $\varepsilon(\mathbf{x})$ and magnetic permeability $\mu(\mathbf{x})$ such that

$$
\begin{aligned}
& \varepsilon(\mathbf{x})= \begin{cases}\varepsilon_{m} & \text { for } \mathbf{x} \in \Sigma_{m} \\
\varepsilon_{s} & \text { for } \mathbf{x} \in \Delta_{s} \\
\varepsilon_{0} & \text { for } \mathbf{x} \in \mathbb{R}^{2} \backslash(\bar{\Sigma} \cup \bar{\Delta}),\end{cases} \\
& \mu(\mathbf{x})= \begin{cases}\mu_{m} & \text { for } \mathbf{x} \in \Sigma_{m} \\
\mu_{s} & \text { for } \mathbf{x} \in \Delta_{s} \\
\mu_{0} & \text { for } \mathbf{x} \in \mathbb{R}^{2} \backslash(\bar{\Sigma} \cup \bar{\Delta}),\end{cases}
\end{aligned}
$$

respectively. For the sake of simplicity, we let $\varepsilon_{0}=\mu_{0}=1$, $\varepsilon_{m}>\varepsilon_{s}$, and $\mu_{m}>\mu_{s}$ for all $m$ and $s$. Hence, we can set the wavenumber $k=\omega \sqrt{\varepsilon_{0} \mu_{0}}=\omega$.
For a given fixed frequency $\omega$, we denote

$$
u_{\text {inc }}(\mathbf{x}, \boldsymbol{\theta})=e^{i \omega \boldsymbol{\theta} \cdot \mathbf{x}}
$$

to be a plane-wave incident field with the incident direction $\boldsymbol{\theta} \in \mathbb{S}^{1}$, where $\mathbb{S}^{1}$ denotes the two-dimensional unit circle. Let $u(\mathbf{x}, \boldsymbol{\theta})$ denote the time-harmonic total field that satisfies the following Helmholtz equation

$$
\nabla \cdot\left(\frac{1}{\mu(\mathbf{x})} \nabla u(\mathbf{x}, \boldsymbol{\theta})\right)+\omega^{2} \varepsilon(\mathbf{x}) u(\mathbf{x}, \boldsymbol{\theta})=0
$$

with transmission conditions on the boundaries of $\Sigma_{m}$ and $\Delta_{s}$. This configuration is associated with a scalar scattering problem for $E$-polarized (Transverse Magnetic (TM) polarization, corresponding to dielectric contrasts) field; the $H$ polarized (Transverse Electric (TE) polarization, corresponding to magnetic contrasts) case could be dealt with per duality. It is well known that $u(\mathbf{x}, \boldsymbol{\theta})$ can be decomposed as

$$
u(\mathbf{x}, \boldsymbol{\theta})=u_{\text {inc }}(\mathbf{x}, \boldsymbol{\theta})+u_{\text {scat }}(\mathbf{x}, \boldsymbol{\theta}),
$$

where $u_{\text {scat }}(\mathbf{x}, \boldsymbol{\theta})$ denotes the unknown scattered field that satisfies the Sommerfeld radiation condition

$$
\lim _{|\mathbf{x}| \rightarrow 0} \sqrt{|\mathbf{x}|}\left(\frac{\partial u_{\text {scat }}(\mathbf{x}, \boldsymbol{\theta})}{\partial|\mathbf{x}|}-i \omega u_{\text {scat }}(\mathbf{x}, \boldsymbol{\theta})\right)=0
$$

uniformly in all directions $\boldsymbol{\vartheta}=\mathbf{x} /|\mathbf{x}| \in \mathbb{S}^{1}$. The far-field pattern $u_{\text {far }}(\boldsymbol{\theta}, \boldsymbol{\theta})$ of the scattered field $u_{\text {scat }}(\mathbf{x}, \boldsymbol{\theta})$ is defined on $\mathbb{S}^{1}$. It can be expressed as

$$
u_{\text {scat }}(\mathbf{x}, \boldsymbol{\theta})=\frac{e^{i \omega|\mathbf{x}|}}{\sqrt{|\mathbf{x}|}} u_{\text {far }}(\boldsymbol{\vartheta}, \boldsymbol{\theta})+o\left(\frac{1}{\sqrt{|\mathbf{x}|}}\right),
$$

$$
|\mathbf{x}| \longrightarrow+\infty
$$

Then by virtue of [31], the far-field pattern $u_{\mathrm{far}}(\boldsymbol{\vartheta}, \boldsymbol{\theta})$ can be written as the following asymptotic expansion formula, which plays a key role in the MUSIC-type algorithm that will be designed in the next section:

$$
\begin{aligned}
u_{\mathrm{far}} & (\boldsymbol{\vartheta}, \boldsymbol{\theta})=\frac{\omega^{2}(1+i)}{4 \sqrt{\omega \pi}}\left\{\sum _ { m = 1 } ^ { M } r _ { m } ^ { 2 } \left(\left(\varepsilon_{m}-\varepsilon_{0}\right)\left|\mathbf{B}_{m}\right|\right.\right. \\
& \left.-\frac{\mu_{0}}{\mu_{m}+\mu_{0}}(\sqrt{2} \boldsymbol{\vartheta}) \cdot(\sqrt{2} \boldsymbol{\theta})\right) e^{i \omega(\boldsymbol{\theta}-\mathbf{9}) \cdot \mathbf{z}_{m}} \\
& +\sum_{s=1}^{S} r_{s}^{2}\left(\frac{\varepsilon_{s}-\varepsilon_{0}}{\sqrt{\varepsilon_{0} \mu_{0}}}\left|\mathbf{B}_{s}\right|-\frac{\mu_{0}}{\mu_{s}+\mu_{0}}(\sqrt{2} \boldsymbol{\vartheta}) \cdot(\sqrt{2} \boldsymbol{\theta})\right) \\
\cdot & \left.e^{i \omega(\boldsymbol{\theta}-\mathbf{9}) \cdot \mathbf{y}_{s}}\right\} .
\end{aligned}
$$

\section{MUSIC-Type Imaging Algorithm}

In this section, we introduce the MUSIC-type algorithm for detecting the locations of small inhomogeneities. For the sake of simplicity, we exclude the constant term $\omega^{2}(1+i) / 4 \sqrt{\omega \pi}$ 
from (10). For this, let us consider the eigenvalue structure of the MSR matrix

$\mathbb{K}$

$$
=\left[\begin{array}{cccc}
u_{\mathrm{far}}\left(\boldsymbol{\vartheta}_{1}, \boldsymbol{\theta}_{1}\right) & u_{\mathrm{far}}\left(\boldsymbol{\vartheta}_{1}, \boldsymbol{\theta}_{2}\right) & \cdots & u_{\mathrm{far}}\left(\boldsymbol{\vartheta}_{1}, \boldsymbol{\theta}_{N}\right) \\
u_{\mathrm{far}}\left(\boldsymbol{\vartheta}_{2}, \boldsymbol{\theta}_{1}\right) & u_{\mathrm{far}}\left(\boldsymbol{\vartheta}_{2}, \boldsymbol{\theta}_{2}\right) & \cdots & u_{\mathrm{far}}\left(\boldsymbol{\vartheta}_{2}, \boldsymbol{\theta}_{N}\right) \\
\vdots & \vdots & \ddots & \vdots \\
u_{\mathrm{far}}\left(\boldsymbol{\vartheta}_{N}, \boldsymbol{\theta}_{1}\right) & u_{\mathrm{far}}\left(\boldsymbol{\vartheta}_{N}, \boldsymbol{\theta}_{2}\right) & \cdots & u_{\mathrm{far}}\left(\boldsymbol{\vartheta}_{N}, \boldsymbol{\theta}_{N}\right)
\end{array}\right] \text {. }
$$

Suppose that $\boldsymbol{\vartheta}_{j}=-\boldsymbol{\theta}_{j}$ for all $\boldsymbol{j}$; then $\mathbb{K}$ is a complex symmetric matrix but not a Hermitian. Thus, instead of eigenvalue decomposition, we perform singular value decomposition (SVD) of $\mathbb{K}$ (see [24], for instance)

$$
\mathbb{K} \approx \sum_{m=1}^{3 M} \sigma_{m} \mathbf{U}_{m} \mathbf{V}_{m}^{*}+\sum_{s=3 M+1}^{3 M+3 S} \sigma_{s} \mathbf{U}_{s} \mathbf{V}_{s}^{*},
$$

where superscript $*$ is the mark of a Hermitian. Then, $\left\{\mathbf{U}_{1}, \mathbf{U}_{2}, \ldots, \mathbf{U}_{3 M+3 S}\right\}$ is the orthogonal basis for the signal space of $\mathbb{K}$. Therefore, one can define the projection operator onto the null (or noise) subspace, $\mathbf{P}_{\text {noise }}: \mathbb{C}^{N \times 1} \rightarrow \mathbb{C}^{N \times 1}$. This projection is given explicitly by

$$
\mathbf{P}_{\text {noise }}:=\mathbb{\square}_{N}-\sum_{m=1}^{3 M+3 S} \mathbf{U}_{m} \mathbf{U}_{m}^{*},
$$

where $\mathbb{q}_{N}$ denotes the $N \times N$ identity matrix. For any point $\mathbf{x} \in \mathbb{R}^{2}$ and suitable vectors $\mathbf{c}_{n} \in \mathbb{R}^{3} \backslash\{\mathbf{0}\}, n=1,2, \ldots, N$, define a test vector $\mathbf{f}(\mathbf{x}) \in \mathbb{C}^{N \times 1}$ as

$$
\begin{aligned}
& \mathbf{f}(\mathbf{x})=\left[\mathbf{c}_{1} \cdot\left[1, \boldsymbol{\theta}_{1}\right]^{T} e^{i \omega \boldsymbol{\theta}_{1} \cdot \mathbf{x}}, \mathbf{c}_{2} \cdot\left[1, \boldsymbol{\theta}_{2}\right]^{T} e^{i \omega \boldsymbol{\theta}_{2} \cdot \mathbf{x}}, \ldots, \mathbf{c}_{N}\right. \\
&\left.\cdot\left[1, \boldsymbol{\theta}_{N}\right]^{T} e^{i \omega \boldsymbol{\theta}_{N} \cdot \mathbf{x}}\right]^{T} .
\end{aligned}
$$

Then, by virtue of [23], there exists $N_{0} \in \mathbb{N}$ such that, for any $N \geq N_{0}$, the following statement holds:

$$
\mathbf{f}(\mathbf{x}) \in \operatorname{Range}(\mathbb{K} \overline{\mathbb{K}}) \quad \text { iff } \mathbf{x} \in\left\{\mathbf{z}_{m}, \mathbf{y}_{s}\right\}
$$

for $m=1,2, \ldots, M$ and $s=1,2, \ldots, S$. This means that if $\mathbf{x} \in \Sigma_{m}$ or $\mathbf{x} \in \Delta_{s}$ then $\left|\mathbf{P}_{\text {noise }}(\mathbf{f}(\mathbf{x}))\right|=0$. Thus, the locations of $\Sigma_{m}$ and $\Delta_{s}$ follow from computing the MUSIC-type imaging function

$$
\mathscr{F}(\mathbf{x})=\frac{1}{\left|\mathbf{P}_{\text {noise }}(\mathbf{f}(\mathbf{x}))\right|} .
$$

The resulting plot of $\mathscr{F}(\mathbf{x})$ will have peaks of large magnitudes at $\mathbf{z}_{m} \in \Sigma_{m}$ and $\mathbf{y}_{s} \in \Delta_{s}$.

Remark 1. Based on several works $[17,18,20]$, selection of $\mathbf{c}_{n}$ in (14) is highly depending on the shape of $\Sigma_{m}$. Unfortunately, the shape of $\Sigma_{m}$ is unknown; it is impossible to find proper vectors $c_{n}$. Due to this fact, following [20], we assume that $\mathbf{c}_{n} \cdot\left[1, \boldsymbol{\theta}_{n}\right]^{T}=1$ for all $n$; that is, we consider the following test vector instead of (14):

$$
\mathbf{f}(\mathbf{x})=\frac{1}{\sqrt{N}}\left[e^{i \omega \boldsymbol{\theta}_{1} \cdot \mathbf{x}}, e^{i \omega \boldsymbol{\theta}_{2} \cdot \mathbf{x}}, \ldots, e^{i \omega \boldsymbol{\theta}_{N} \cdot \mathbf{x}}\right]^{T}
$$

and we analyze the mathematical structure of $\mathscr{F}(\mathbf{x})$.

\section{Structure of Imaging Function}

Henceforth, we analyze the mathematical structure of $\mathscr{F}(\mathbf{x})$ and examine certain of its properties. Before starting, we recall a useful result derived in [32].

Lemma 2. Assume that $\left\{\boldsymbol{\theta}_{n}: n=1,2, \ldots, N\right\}$ spans $\mathbb{S}^{1}$. Then, for sufficiently large $N, \boldsymbol{\xi} \in \mathbb{S}^{1}$, and $\mathbf{x} \in \mathbb{R}^{2}$, the following relation holds:

$$
\begin{aligned}
\frac{1}{N} \sum_{n=1}^{N} e^{i \omega \boldsymbol{\theta}_{n} \cdot \mathbf{x}} & =\frac{1}{2 \pi} \int_{\mathbb{S}^{1}} e^{i \omega \boldsymbol{\theta} \cdot \mathbf{x}} d S(\boldsymbol{\theta}) \\
& =J_{0}(\omega|\mathbf{x}|), \\
\frac{1}{N} \sum_{n=1}^{N}\left(\boldsymbol{\xi} \cdot \boldsymbol{\theta}_{n}\right) e^{i \omega \boldsymbol{\theta}_{n} \cdot \mathbf{x}} & =\frac{1}{2 \pi} \int_{\mathbb{S}^{1}}(\boldsymbol{\xi} \cdot \boldsymbol{\theta}) e^{i \omega \boldsymbol{\theta} \cdot \mathbf{x}} d S(\boldsymbol{\theta}) \\
& =i\left(\frac{\mathbf{x}}{|\mathbf{x}|} \cdot \boldsymbol{\xi}\right) J_{1}(\omega|\mathbf{x}|),
\end{aligned}
$$

where $J_{n}$ denotes Bessel function of order $n$ of the first kind.

Now, we introduce the main result.

Theorem 3. For sufficiently large $N>3 M+3 S$ and $\omega, \mathscr{F}(\mathbf{x})$ can be represented as follows: for $\mathbf{e}_{1}=[1,0]^{T}$ and $\mathbf{e}_{2}=[0,1]^{T}$,

$$
\begin{aligned}
\mathscr{F}(\mathbf{x}) & \approx\left(1-\sum_{m=1}^{M} J_{0}\left(\omega\left|\mathbf{x}-\mathbf{z}_{m}\right|\right)^{2}\right. \\
& -\sum_{m=1}^{M} \sum_{h=1}^{2}\left(\frac{\left(\mathbf{x}-\mathbf{z}_{m}\right) \cdot \mathbf{e}_{h}}{\left|\mathbf{x}-\mathbf{z}_{m}\right|}\right)^{2} J_{1}\left(\omega\left|\mathbf{x}-\mathbf{z}_{m}\right|\right)^{2} \\
& -\sum_{s=1}^{S} J_{0}\left(\omega\left|\mathbf{x}-\mathbf{y}_{s}\right|\right)^{2} \\
& \left.-\sum_{s=1}^{S} \sum_{h=1}^{2}\left(\frac{\left(\mathbf{x}-\mathbf{y}_{s}\right) \cdot \mathbf{e}_{h}}{\left|\mathbf{x}-\mathbf{y}_{s}\right|}\right)^{2} J_{1}\left(\omega\left|\mathbf{x}-\mathbf{y}_{s}\right|\right)^{2}\right)^{-1 / 2} .
\end{aligned}
$$

Proof. Based on the asymptotic expansion formula (10) and results in [13], $\mathbf{P}_{\text {noise }}$ can be represented as

$$
\begin{aligned}
\mathbf{P}_{\text {noise }}= & \mathbb{q}_{N}-\sum_{m=1}^{3 M} \mathbf{U}_{m} \mathbf{U}_{m}^{*}-\sum_{s=1}^{3 S} \mathbf{U}_{s} \mathbf{U}_{s}^{*} \\
\approx & \mathbb{\square}_{N}-\sum_{m=1}^{M} \sum_{h=1}^{3} \mathbf{W}_{h}\left(\mathbf{z}_{m}\right) \mathbf{W}_{h}\left(\mathbf{z}_{m}\right)^{*} \\
& -\sum_{s=1}^{S} \sum_{h=1}^{3} \mathbf{W}_{h}\left(\mathbf{y}_{s}\right) \mathbf{W}_{h}\left(\mathbf{y}_{s}\right)^{*}
\end{aligned}
$$

where

$$
\begin{gathered}
\mathbf{W}_{1}(\mathbf{x})=\frac{1}{\sqrt{N}}\left[e^{i \omega \boldsymbol{\theta}_{1} \cdot \mathbf{x}}, e^{i \omega \boldsymbol{\theta}_{2} \cdot \mathbf{x}}, \ldots, e^{i \omega \boldsymbol{\theta}_{N} \cdot \mathbf{x}}\right]^{T}, \\
\mathbf{W}_{2}(\mathbf{x})=\frac{\sqrt{2}}{\sqrt{N}}\left[\left(\mathbf{e}_{1} \cdot \boldsymbol{\theta}_{1}\right) e^{i \omega \boldsymbol{\theta}_{1} \cdot \mathbf{x}},\left(\mathbf{e}_{1} \cdot \boldsymbol{\theta}_{2}\right)\right. \\
\left.\cdot e^{i \omega \boldsymbol{\theta}_{2} \cdot \mathbf{x}}, \ldots,\left(\mathbf{e}_{1} \cdot \boldsymbol{\theta}_{N}\right) e^{i \omega \boldsymbol{\theta}_{N} \cdot \mathbf{x}}\right]^{T},
\end{gathered}
$$




$$
\begin{gathered}
\mathbf{W}_{3}(\mathbf{x})=\frac{\sqrt{2}}{\sqrt{N}}\left[\left(\mathbf{e}_{2} \cdot \boldsymbol{\theta}_{1}\right) e^{i \omega \boldsymbol{\theta}_{1} \cdot \mathbf{x}},\left(\mathbf{e}_{2} \cdot \boldsymbol{\theta}_{2}\right)\right. \\
\left.\cdot e^{i \omega \boldsymbol{\theta}_{2} \cdot \mathbf{x}}, \ldots,\left(\mathbf{e}_{2} \cdot \boldsymbol{\theta}_{N}\right) e^{i \omega \boldsymbol{\theta}_{N} \cdot \mathbf{x}}\right]^{T}
\end{gathered}
$$

With this, applying (18) and performing a tedious calculation, we arrive at

$$
\begin{aligned}
\mathbf{P}_{\text {noise }}(\mathbf{f}(\mathbf{x}))=\mathbf{f}(\mathbf{x}) & \\
- & \frac{1}{N \sqrt{N}}\left(\sum_{m=1}^{M}\left(\mathbb{A}\left(\mathbf{z}_{m}\right)+\mathbb{B}_{1}\left(\mathbf{z}_{m}\right)+\mathbb{B}_{2}\left(\mathbf{z}_{m}\right)\right)\right. \\
& \left.-\sum_{s=1}^{S}\left(\mathbb{A}\left(\mathbf{y}_{s}\right)+\mathbb{B}_{1}\left(\mathbf{y}_{s}\right)+\mathbb{B}_{2}\left(\mathbf{y}_{s}\right)\right)\right),
\end{aligned}
$$

where

$$
\mathbb{A}(\boldsymbol{\xi}):=\left[\begin{array}{c}
e^{i \omega \boldsymbol{\theta}_{1} \cdot \xi} J_{0}(\omega|\mathbf{x}-\boldsymbol{\xi}|) \\
e^{i \omega \boldsymbol{\theta}_{2} \cdot \xi} J_{0}(\omega|\mathbf{x}-\boldsymbol{\xi}|) \\
\vdots \\
e^{i \omega \boldsymbol{\theta}_{N} \cdot \xi} J_{0}(\omega|\mathbf{x}-\boldsymbol{\xi}|)
\end{array}\right],
$$

$\mathbb{B}_{h}(\xi)$

$$
:=\left[\begin{array}{c}
i\left(\mathbf{e}_{h} \cdot \boldsymbol{\theta}_{1}\right)\left(\frac{(\mathbf{x}-\boldsymbol{\xi}) \cdot \mathbf{e}_{h}}{|\mathbf{x}-\boldsymbol{\xi}|}\right) e^{i \omega \boldsymbol{\theta}_{1} \cdot \xi} J_{1}(\omega|\mathbf{x}-\boldsymbol{\xi}|) \\
i\left(\mathbf{e}_{h} \cdot \boldsymbol{\theta}_{2}\right)\left(\frac{(\mathbf{x}-\boldsymbol{\xi}) \cdot \mathbf{e}_{h}}{|\mathbf{x}-\boldsymbol{\xi}|}\right) e^{i \omega \boldsymbol{\theta}_{2} \cdot \xi} J_{1}(\omega|\mathbf{x}-\boldsymbol{\xi}|) \\
\vdots \\
i\left(\mathbf{e}_{h} \cdot \boldsymbol{\theta}_{N}\right)\left(\frac{(\mathbf{x}-\boldsymbol{\xi}) \cdot \mathbf{e}_{h}}{|\mathbf{x}-\boldsymbol{\xi}|}\right) e^{i \omega \boldsymbol{\theta}_{N} \cdot \xi} J_{1}(\omega|\mathbf{x}-\boldsymbol{\xi}|)
\end{array}\right]
$$

for $\xi \in \mathbb{R}^{2}$ and $h=1,2$. By implementing elementary calculus, we can show that

$$
\begin{aligned}
\left|\mathbf{P}_{\text {noise }}(\mathbf{f}(\mathbf{x}))\right|^{2} & =\mathbf{P}_{\text {noise }}(\mathbf{f}(\mathbf{x})) \overline{\mathbf{P}_{\text {noise }}(\mathbf{f}(\mathbf{x}))} \\
& =\frac{1}{N} \sum_{n=1}^{N}\left(1-\sum_{h=1}^{8} \Phi_{h}\right)
\end{aligned}
$$

where

$$
\begin{aligned}
\Phi_{1} & =\sum_{m=1}^{M}\left(e^{i \omega \boldsymbol{\theta}_{n} \cdot\left(\mathbf{x}-\mathbf{z}_{m}\right)}+e^{-i \omega \boldsymbol{\theta}_{n} \cdot\left(\mathbf{x}-\mathbf{z}_{m}\right)}\right) J_{0}\left(\omega\left|\mathbf{x}-\mathbf{z}_{m}\right|\right), \\
\Phi_{2} & =-\left(\sum_{m=1}^{M} e^{i \omega \boldsymbol{\theta}_{n^{\prime}} \cdot \mathbf{z}_{m}} J_{0}\left(\omega\left|\mathbf{x}-\mathbf{z}_{m}\right|\right)\right) \\
& \cdot\left(\sum_{m^{\prime}=1}^{M} e^{-i \omega \boldsymbol{\theta}_{n} \cdot \mathbf{z}_{m^{\prime}}} J_{0}\left(\omega\left|\mathbf{x}-\mathbf{z}_{m^{\prime}}\right|\right)\right)
\end{aligned}
$$

$$
\begin{aligned}
& \Phi_{3}=-i \sum_{m=1}^{M} \sum_{h=1}^{2}\left(\mathbf{e}_{h} \cdot \boldsymbol{\theta}_{n}\right)\left(\frac{\left(\mathbf{x}-\mathbf{z}_{m}\right) \cdot \mathbf{e}_{h}}{\left|\mathbf{x}-\mathbf{z}_{m}\right|}\right)\left(e^{i \omega \boldsymbol{\theta}_{n} \cdot\left(\mathbf{x}-\mathbf{z}_{m}\right)}\right. \\
& \left.-e^{-i \omega \boldsymbol{\theta}_{n} \cdot\left(\mathbf{x}-\mathbf{z}_{m}\right)}\right) J_{1}\left(\omega\left|\mathbf{x}-\mathbf{z}_{m}\right|\right), \\
& \Phi_{4}=-\left(\sum_{m=1}^{M} \sum_{h=1}^{2}\left(\mathbf{e}_{h} \cdot \boldsymbol{\theta}_{n}\right)\left(\frac{\left(\mathbf{x}-\mathbf{z}_{m}\right) \cdot \mathbf{e}_{h}}{\left|\mathbf{x}-\mathbf{z}_{m}\right|}\right)\right. \\
& \left.\cdot e^{i \omega \boldsymbol{\theta}_{n} \cdot \mathbf{z}_{m}} J_{1}\left(\omega\left|\mathbf{x}-\mathbf{z}_{m}\right|\right)\right)\left(\sum_{m^{\prime}=1}^{M} \sum_{h=1}^{2}\left(\mathbf{e}_{h} \cdot \boldsymbol{\theta}_{n}\right)\right.
\end{aligned}
$$

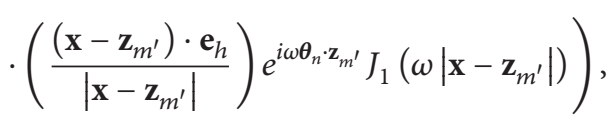

$$
\begin{aligned}
& \Phi_{5}=\sum_{s=1}^{S}\left(e^{i \omega \boldsymbol{\theta}_{n} \cdot\left(\mathbf{x}-\mathbf{y}_{s}\right)}+e^{-i \omega \boldsymbol{\theta}_{n} \cdot\left(\mathbf{x}-\mathbf{y}_{s}\right)}\right) J_{0}\left(\omega\left|\mathbf{x}-\mathbf{y}_{s}\right|\right), \\
& \Phi_{6}=-\left(\sum_{s=1}^{S} e^{i \omega \boldsymbol{\theta}_{n} \cdot \mathbf{y}_{s}} J_{0}\left(\omega\left|\mathbf{x}-\mathbf{y}_{s}\right|\right)\right) \\
& \cdot\left(\sum_{s^{\prime}=1}^{S} e^{-i \omega \boldsymbol{\theta}_{n} \cdot \mathbf{y}_{s^{\prime}}} J_{0}\left(\omega\left|\mathbf{x}-\mathbf{y}_{s^{\prime}}\right|\right)\right), \\
& \Phi_{7}=-i \sum_{s=1}^{M} \sum_{h=1}^{2}\left(\mathbf{e}_{h} \cdot \boldsymbol{\theta}_{n}\right)\left(\frac{\left(\mathbf{x}-\mathbf{y}_{s}\right) \cdot \mathbf{e}_{h}}{\left|\mathbf{x}-\mathbf{y}_{s}\right|}\right)\left(e^{i \omega \boldsymbol{\theta}_{n} \cdot\left(\mathbf{x}-\mathbf{y}_{s}\right)}\right. \\
& \left.+e^{-i \omega \boldsymbol{\theta}_{n} \cdot\left(\mathbf{x}-\mathbf{y}_{s}\right)}\right) J_{1}\left(\omega\left|\mathbf{x}-\mathbf{y}_{s}\right|\right), \\
& \Phi_{8}=-\left(\sum_{s=1}^{M} \sum_{h=1}^{2}\left(\mathbf{e}_{h} \cdot \boldsymbol{\theta}_{n}\right)\left(\frac{\left(\mathbf{x}-\mathbf{y}_{s}\right) \cdot \mathbf{e}_{h}}{\left|\mathbf{x}-\mathbf{y}_{s}\right|}\right)\right. \\
& \left.\cdot e^{i \omega \boldsymbol{\theta}_{n} \cdot \mathbf{y}_{s}} J_{1}\left(\omega\left|\mathbf{x}-\mathbf{y}_{s}\right|\right)\right)\left(\sum_{s^{\prime}=1}^{M} \sum_{h=1}^{2}\left(\mathbf{e}_{h} \cdot \boldsymbol{\theta}_{n}\right)\right. \\
& \left.\cdot\left(\frac{\left(\mathbf{x}-\mathbf{z}_{s^{\prime}}\right) \cdot \mathbf{e}_{h}}{\left|\mathbf{x}-\mathbf{z}_{s^{\prime}}\right|}\right) e^{i \omega \boldsymbol{\theta}_{n^{\prime}} \cdot \mathbf{z}_{s^{\prime}}} J_{1}\left(\omega\left|\mathbf{x}-\mathbf{z}_{s^{\prime}}\right|\right)\right) .
\end{aligned}
$$

First, applying (18), we can obtain

$$
\begin{gathered}
\frac{1}{N} \sum_{n=1}^{N} \sum_{m=1}^{M} e^{i \omega \boldsymbol{\theta}_{n} \cdot\left(\mathbf{x}-\mathbf{z}_{m}\right)} J_{0}\left(\omega\left|\mathbf{x}-\mathbf{z}_{m}\right|\right) \\
\quad=\sum_{m=1}^{M} J_{0}\left(\omega\left|\mathbf{x}-\mathbf{z}_{m}\right|\right)^{2} .
\end{gathered}
$$

This leads us to

$$
\begin{aligned}
& \frac{1}{N} \sum_{n=1}^{N} \Phi_{1}=\frac{1}{N} \\
& \quad \cdot \sum_{n=1}^{N} \sum_{m=1}^{M}\left(e^{i \omega \boldsymbol{\theta}_{n} \cdot\left(\mathbf{x}-\mathbf{z}_{m}\right)}+e^{-i \omega \boldsymbol{\theta}_{n} \cdot\left(\mathbf{x}-\mathbf{z}_{m}\right)}\right) J_{0}\left(\omega\left|\mathbf{x}-\mathbf{z}_{m}\right|\right) \\
& \quad=2 \sum_{m=1}^{M} J_{0}\left(\omega\left|\mathbf{x}-\mathbf{z}_{m}\right|\right)^{2}
\end{aligned}
$$


and similarly to

$$
\begin{aligned}
& \frac{1}{N} \sum_{n=1}^{N} \Phi_{5} \\
& \quad=\frac{1}{N} \sum_{n=1}^{N} \sum_{s=1}^{S}\left(e^{i \omega \boldsymbol{\theta}_{n} \cdot\left(\mathbf{x}-\mathbf{y}_{s}\right)}+e^{-i \omega \boldsymbol{\theta}_{n} \cdot\left(\mathbf{x}-\mathbf{y}_{s}\right)}\right) J_{0}\left(\omega\left|\mathbf{x}-\mathbf{y}_{s}\right|\right) \\
& \quad=2 \sum_{s=1}^{S} J_{0}\left(\omega\left|\mathbf{x}-\mathbf{y}_{s}\right|\right)^{2}
\end{aligned}
$$

Next, based on the orthonormal property of singular vectors, relations (2) and (18), and the following asymptotic form

$$
\begin{aligned}
& J_{0}\left(\omega\left|\mathbf{z}-\mathbf{z}_{m^{\prime}}\right|\right) \\
& \quad \approx \sqrt{\frac{2}{\omega\left|\mathbf{z}-\mathbf{z}_{m^{\prime}}\right| \pi}} \cos \left(\omega\left|\mathbf{z}-\mathbf{z}_{m^{\prime}}\right|-\frac{\pi}{4}\right),
\end{aligned}
$$

we can derive

$$
\begin{aligned}
& \frac{1}{N} \sum_{n=1}^{N} \Phi_{2}=-\frac{1}{N} \sum_{n=1}^{N}\left(\sum_{m=1}^{M} e^{i \omega \boldsymbol{\theta}_{n} \cdot \mathbf{z}_{m}} J_{0}\left(\omega\left|\mathbf{x}-\mathbf{z}_{m}\right|\right)\right) \\
& \cdot\left(\sum_{m^{\prime}=1}^{M} e^{-i \omega \boldsymbol{\theta}_{n} \cdot \mathbf{z}_{m^{\prime}}} J_{0}\left(\omega\left|\mathbf{x}-\mathbf{z}_{m^{\prime}}\right|\right)\right) \\
& =-\sum_{m=1}^{M} \sum_{m^{\prime}=1}^{M}\left(\frac{1}{N}\right. \\
& \left.\cdot \sum_{n=1}^{N} e^{i \omega \boldsymbol{\theta}_{n} \cdot\left(\mathbf{z}-\mathbf{z}_{m^{\prime}}\right)} J_{0}\left(\omega\left|\mathbf{x}-\mathbf{z}_{m}\right|\right) J_{0}\left(\omega\left|\mathbf{x}-\mathbf{z}_{m^{\prime}}\right|\right)\right) \\
& =-\sum_{m=1}^{M} \sum_{m^{\prime}=1}^{M} J_{0}\left(\omega\left|\mathbf{z}-\mathbf{z}_{m^{\prime}}\right|\right) J_{0}\left(\omega\left|\mathbf{x}-\mathbf{z}_{m}\right|\right) \\
& \cdot J_{0}\left(\omega\left|\mathbf{x}-\mathbf{z}_{m^{\prime}}\right|\right)=-\sum_{m=1}^{M} J_{0}\left(\omega\left|\mathbf{x}-\mathbf{z}_{m}\right|\right)^{2}
\end{aligned}
$$

and similarly

$$
\frac{1}{N} \sum_{n=1}^{N} \Phi_{6}=-\sum_{s=1}^{S} J_{0}\left(\omega\left|\mathbf{x}-\mathbf{y}_{s}\right|\right)^{2}
$$

For evaluating $\Phi_{3}$, let us perform an elementary calculus

$$
\begin{gathered}
\frac{1}{N} \sum_{n=1}^{N}\left(i \sum_{m=1}^{M} \sum_{h=1}^{2}\left(\mathbf{e}_{h} \cdot \boldsymbol{\theta}_{n}\right) e^{i \omega \boldsymbol{\theta}_{n} \cdot\left(\mathbf{x}-\mathbf{z}_{m}\right)}\right)\left(\frac{\left(\mathbf{x}-\mathbf{z}_{m}\right) \cdot \mathbf{e}_{h}}{\left|\mathbf{x}-\mathbf{z}_{m}\right|}\right) \\
\cdot J_{1}\left(\omega\left|\mathbf{x}-\mathbf{z}_{m}\right|\right) \\
=\sum_{m=1}^{M} \sum_{h=1}^{2}\left(i \frac{1}{N} \sum_{n=1}^{N}\left(\mathbf{e}_{h} \cdot \boldsymbol{\theta}_{n}\right) e^{i \omega \boldsymbol{\theta}_{n} \cdot\left(\mathbf{x}-\mathbf{z}_{m}\right)}\right)
\end{gathered}
$$

$$
\begin{aligned}
& \left(\frac{\left(\mathbf{x}-\mathbf{z}_{m}\right) \cdot \mathbf{e}_{h}}{\left|\mathbf{x}-\mathbf{z}_{m}\right|}\right) J_{1}\left(\omega\left|\mathbf{x}-\mathbf{z}_{m}\right|\right) \\
= & -\sum_{m=1}^{M} \sum_{h=1}^{2}\left(\frac{\left(\mathbf{x}-\mathbf{z}_{m}\right) \cdot \mathbf{e}_{h}}{\left|\mathbf{x}-\mathbf{z}_{m}\right|}\right)^{2} J_{1}\left(\omega\left|\mathbf{x}-\mathbf{z}_{m}\right|\right)^{2} .
\end{aligned}
$$

Then, we can conclude that

$$
\begin{aligned}
& \frac{1}{N} \sum_{n=1}^{N} \Phi_{3} \\
& \quad=2 \sum_{m=1}^{M} \sum_{h=1}^{2}\left(\frac{\left(\mathbf{x}-\mathbf{z}_{m}\right) \cdot \mathbf{e}_{h}}{\left|\mathbf{x}-\mathbf{z}_{m}\right|}\right)^{2} J_{1}\left(\omega\left|\mathbf{x}-\mathbf{z}_{m}\right|\right)^{2} \\
& \frac{1}{N} \sum_{n=1}^{N} \Phi_{7}=2 \sum_{s=1}^{S} \sum_{h=1}^{2}\left(\frac{\left(\mathbf{x}-\mathbf{y}_{s}\right) \cdot \mathbf{e}_{h}}{\left|\mathbf{x}-\mathbf{y}_{s}\right|}\right)^{2} J_{1}\left(\omega\left|\mathbf{x}-\mathbf{y}_{s}\right|\right)^{2}
\end{aligned}
$$
$\xi \in \mathbb{S}^{1}$

Finally, for $\Phi_{4}$, by applying following integral, for $\boldsymbol{\theta}_{n}, \boldsymbol{\theta}$,

$$
\frac{1}{N} \sum_{n=1}^{N}\left(\boldsymbol{\theta}_{n} \cdot \boldsymbol{\xi}\right)^{2} \approx \frac{1}{2 \pi} \int_{\mathbb{S}^{1}}(\boldsymbol{\theta} \cdot \boldsymbol{\xi})^{2} d \boldsymbol{\theta}=\frac{1}{2},
$$

we can derive the following:

$$
\begin{aligned}
& \frac{1}{N} \sum_{n=1}^{N} \Phi_{4}=-\frac{1}{N} \\
& \cdot \sum_{n=1}^{N}\left(\sum_{m=1}^{M} \sum_{h=1}^{2}\left(\mathbf{e}_{h} \cdot \boldsymbol{\theta}_{n}\right)\left(\frac{\left(\mathbf{x}-\mathbf{z}_{m}\right) \cdot \mathbf{e}_{h}}{\left|\mathbf{x}-\mathbf{z}_{m}\right|}\right) e^{i \omega \boldsymbol{\theta}_{n} \cdot \mathbf{z}_{m}} J_{1}\left(\omega\left|\mathbf{x}-\mathbf{z}_{m}\right|\right)\right) \\
& \cdot\left(\sum_{m^{\prime}=1}^{M} \sum_{h^{\prime \prime}=1}^{2}\left(\mathbf{e}_{h^{\prime \prime}} \cdot \boldsymbol{\theta}_{n}\right)\left(\frac{\left(\mathbf{x}-\mathbf{z}_{m^{\prime}}\right) \cdot \mathbf{e}_{h^{\prime \prime}}}{\left|\mathbf{x}-\mathbf{z}_{m^{\prime}}\right|}\right)\right. \\
& \left.\cdot e^{i \omega \boldsymbol{\theta}_{n} \cdot \mathbf{z}_{m^{\prime}}} J_{1}\left(\omega\left|\mathbf{x}-\mathbf{z}_{m^{\prime}}\right|\right)\right)=-\sum_{m=1}^{M}\left(\frac{1}{N} \sum_{n=1}^{N} \sum_{h=1}^{2}\left(\mathbf{e}_{s} \cdot \boldsymbol{\theta}_{n}\right)^{2}\right)^{2} \\
& \sum_{h=1}^{2}\left\{\left(\frac{\left(\mathbf{x}-\mathbf{z}_{m}\right) \cdot \mathbf{e}_{s}}{\left|\mathbf{x}-\mathbf{z}_{m}\right|}\right) J_{1}\left(\omega\left|\mathbf{x}-\mathbf{z}_{m}\right|\right)\right\}^{2} \\
& =-\sum_{m=1}^{M} \sum_{h=1}^{2}\left\{\left(\frac{\left(\mathbf{x}-\mathbf{z}_{m}\right) \cdot \mathbf{e}_{h}}{\left|\mathbf{x}-\mathbf{z}_{m}\right|}\right) J_{1}\left(\omega\left|\mathbf{x}-\mathbf{z}_{m}\right|\right)\right\}^{2} .
\end{aligned}
$$

Correspondingly,

$$
\begin{aligned}
& \frac{1}{N} \sum_{n=1}^{N} \Phi_{8} \\
& \quad=-\sum_{s=1}^{S} \sum_{h=1}^{2}\left\{\left(\frac{\left(\mathbf{x}-\mathbf{y}_{s}\right) \cdot \mathbf{e}_{h}}{\left|\mathbf{x}-\mathbf{y}_{s}\right|}\right) J_{1}\left(\omega\left|\mathbf{x}-\mathbf{y}_{s}\right|\right)\right\}^{2} .
\end{aligned}
$$


Hence, by combining (27)-(36), we can obtain the following mathematical structure:

$$
\begin{aligned}
\left|\mathbf{P}_{\text {noise }}(\mathbf{f}(\mathbf{r}))\right|^{2} & \\
= & 1-\sum_{m=1}^{M} J_{0}\left(\omega\left|\mathbf{x}-\mathbf{z}_{m}\right|\right)^{2} \\
& -\sum_{m=1}^{M} \sum_{h=1}^{2}\left(\frac{\left(\mathbf{x}-\mathbf{z}_{m}\right) \cdot \mathbf{e}_{h}}{\left|\mathbf{x}-\mathbf{z}_{m}\right|}\right)^{2} J_{1}\left(\omega\left|\mathbf{x}-\mathbf{z}_{m}\right|\right)^{2} \\
& -\sum_{s=1}^{S} J_{0}\left(\omega\left|\mathbf{x}-\mathbf{y}_{s}\right|\right)^{2} \\
& -\sum_{s=1}^{S} \sum_{h=1}^{2}\left(\frac{\left(\mathbf{x}-\mathbf{y}_{s}\right) \cdot \mathbf{e}_{h}}{\left|\mathbf{x}-\mathbf{y}_{s}\right|}\right)^{2} J_{1}\left(\omega\left|\mathbf{x}-\mathbf{y}_{s}\right|\right)^{2} .
\end{aligned}
$$

This enables us to obtain the desired result. This completes the proof.

Remark 4 (applicability of MUSIC). Since $J_{0}(0)=1$, the value of $\mathscr{F}(\mathbf{x})$ will be sufficiently large when $\mathbf{x}=\mathbf{z}_{m}$ or $\mathbf{y}_{s}$ for all $m$ and $s$. Hence, based on the result in Theorem 3, the locations of $\Sigma_{m}$ and $\Delta_{s}$ can be identified via the map of $\mathscr{F}(\mathbf{z})$. This is the reason why it is possible to detect the locations of small inhomogeneities as well as random scatterers. Note that, for a successful detection, based on the hypothesis in Theorem 3, the value of $N$ (at least, greater than $3 M+3 S$ ) and $\omega$ must be sufficiently large enough. If applied frequency is low or total number of $N$ is small, poor result would appear in the map of $\mathscr{F}(\mathbf{x})$.

Remark 5 (discrimination of singular values). Theoretically, if the size, permittivity, and permeability of the random scatterers are smaller than those of the inhomogeneities, then $\sigma_{s}<\sigma_{m}$ for all $m$ and $s$. This means that if it were possible to discriminate singular values associated with small inhomogeneities, then the structure of $\mathscr{F}(\mathbf{x})$ would become

$$
\begin{aligned}
\mathscr{F}(\mathbf{x}) & \approx\left(1-\sum_{m=1}^{M} J_{0}\left(\omega\left|\mathbf{x}-\mathbf{z}_{m}\right|\right)\right. \\
& \left.-\sum_{m=1}^{M} \sum_{h=1}^{2}\left(\frac{\left(\mathbf{x}-\mathbf{z}_{m}\right) \cdot \mathbf{e}_{h}}{\left|\mathbf{x}-\mathbf{z}_{m}\right|}\right)^{2} J_{1}\left(\omega\left|\mathbf{x}-\mathbf{z}_{m}\right|\right)^{2}\right)^{-1 / 2}
\end{aligned}
$$

Hence, it is expected that more good results can be obtained. Our approach presents an improvement. However, if the relation $\sigma_{s}<\sigma_{m}$ were no longer valid, the locations of random scatterers would have to be identified via MUSIC such that poor results would appear in the map of $\mathscr{F}(\mathbf{x})$.

\section{Results of Numerical Simulations}

Selected results of numerical simulations are presented here to support the identified structure of the MUSIC-type imaging function. In this section, we only consider the dielectric permittivity contrast case; that is, we set $\varepsilon_{m}=3, \varepsilon_{0}=1$, and $\mu_{m}=\mu_{s}=\mu_{0}$ for all $m$ and $s$. The radius of all $\Sigma_{m}$ and $\Delta_{s}$ is set

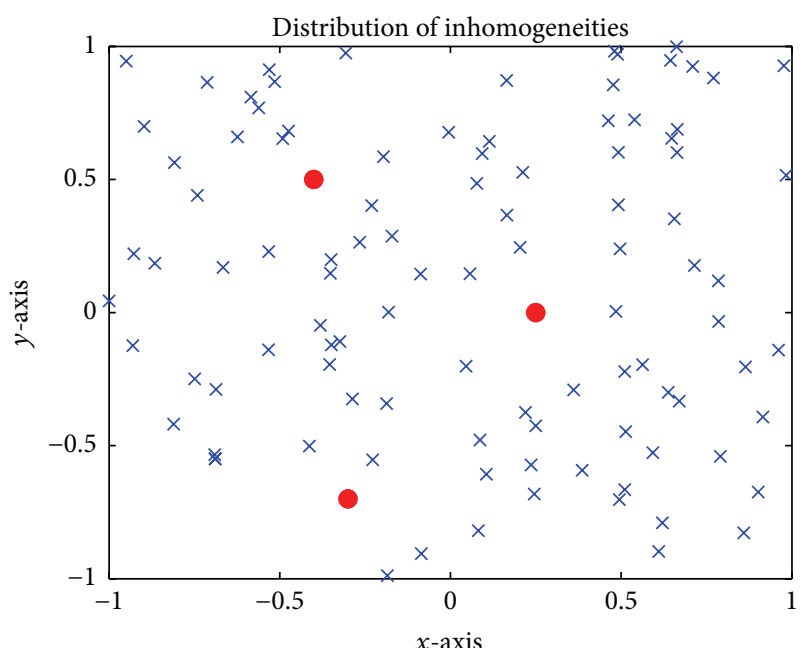

FIGURE 1: Distribution of inhomogeneities (red-colored dots) and random scatterers (blue-colored " $\times$ " mark).

to 0.1 and 0.05 , respectively. The applied angular frequency is $\omega=2 \pi / \lambda$ and a total of $N$ number of incident directions is applied such that

$$
\boldsymbol{\theta}_{j}=-\left[\cos \frac{2 \pi(j-1)}{N}, \frac{2 \pi(j-1)}{N}\right]^{T},{ }_{j=1,2, \ldots, N .}
$$

$M=3$ small inhomogeneities are selected with locations $\mathbf{z}_{1}=[0.25,0]^{T}, \mathbf{z}_{2}=[-0.4,0.5]^{T}$, and $\mathbf{z}_{3}=[-0.3,-0.7]^{T}$. We set $S=100$ number of small scatterers as being randomly distributed in $\Omega=[-1,1] \times[-1,1] \subset \mathbb{R}^{2}$ such that

$$
\mathbf{y}_{s}=\left[\eta_{1}(-1,1), \eta_{2}(-1,1)\right]^{T}
$$

for all $s$ and also select the permittivities randomly as

$$
\varepsilon_{s}=\eta_{3}(1,2) \text {, }
$$

where $\eta_{p}(a, b), p=1,2$, and 3 , is an arbitrary real value within $[a, b]$. Refer to Figure 1 for a sketch of the distribution of the three inhomogeneities and random scatterers.

The far-field elements of MSR matrix $\mathbb{K}$ are generated by means of the Foldy-Lax framework to avoid an inverse crime. After the generation, a singular value decomposition of $\mathbb{K}$ is performed via the MATLAB command svd. The nonzero singular values of $\mathbb{K}$ are discriminated as follows: first, a 0.1 threshold scheme (by first choosing the $j$ singular values $\sigma_{j}$ such that $\sigma_{j} / \sigma_{1} \geq 0.1$ ) is applied based on [18], and second, the first 3-singular values are selected.

Figure 2 exhibits the distribution of the normalized singular values of $\mathbb{K}$ and maps of $\mathscr{F}(\mathbf{x})$ with the 0.1 -threshold scheme and with selection of the first 3-singular values when $\lambda=0.3$ and $N=32$. Note that due to the huge number of artifacts it is very hard to identify the locations of $\Sigma_{m}$ with the 0.1-threshold scheme but, fortunately in this example, one can discriminate three nonzero singular values such that, based on Remark 5, the locations of $\Sigma_{m}$ can be identified 


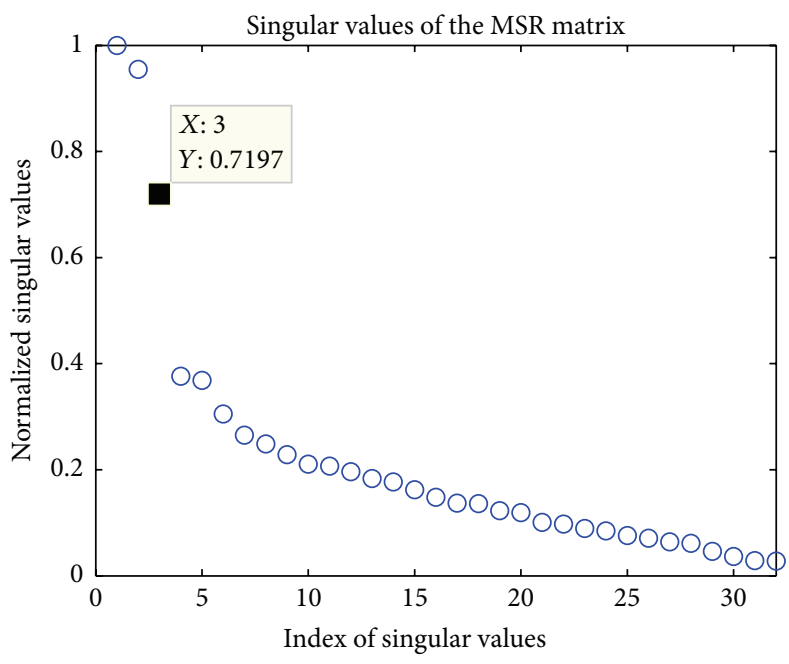

(a)

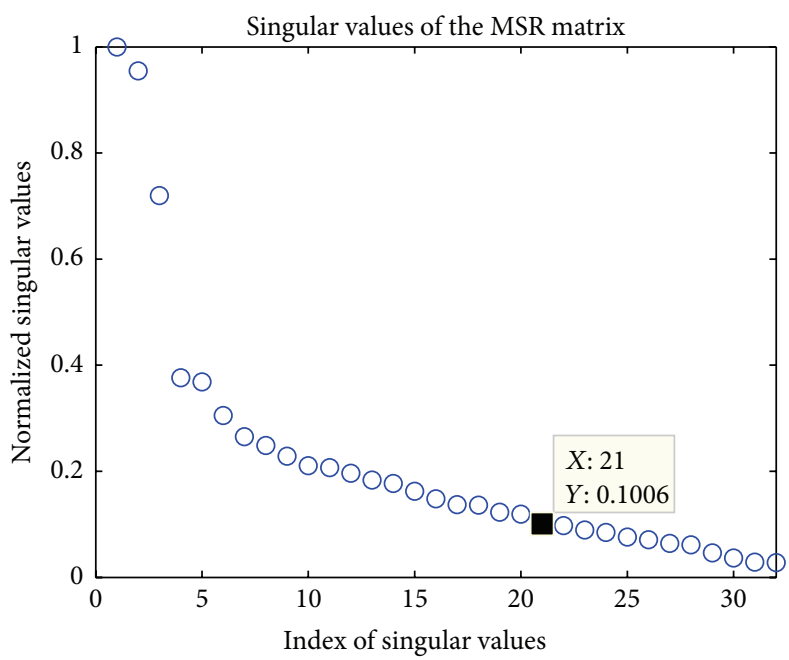

(c)

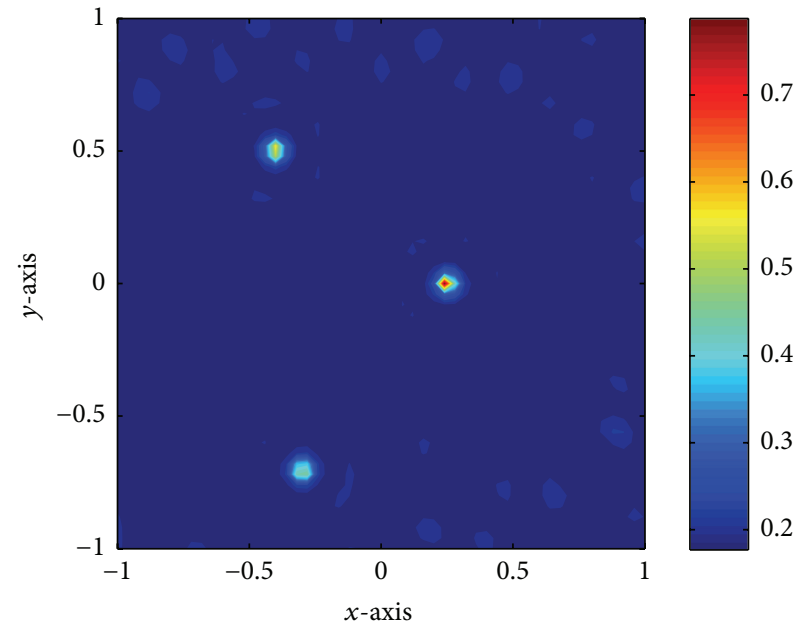

(b)

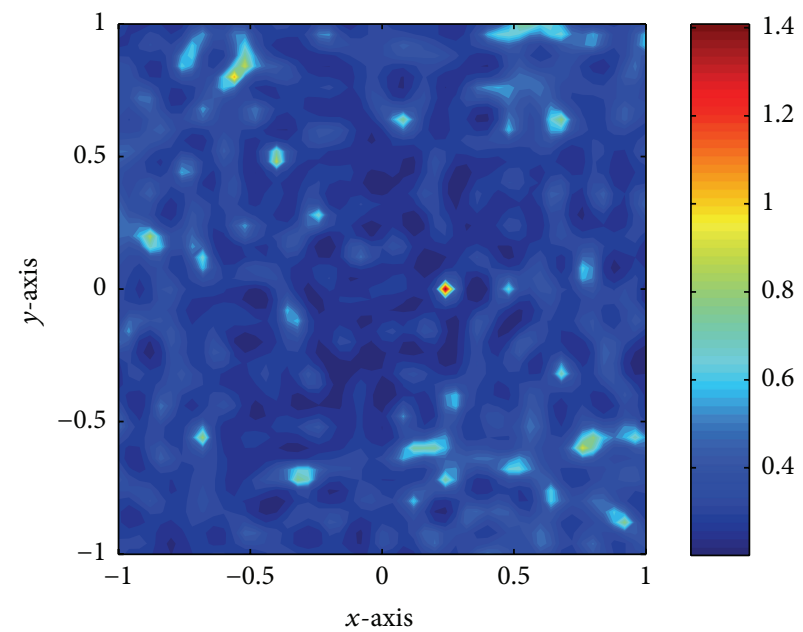

(d)

Figure 2: Distribution of normalized singular values $(\mathrm{a}, \mathrm{c})$ and maps of $\mathscr{F}(\mathbf{x})$ with first 3 -singular values (b) and with 0.1 -threshold scheme (d).

more clearly. This result supports the derived mathematical structure in Theorem 3.

Now, let us examine the effect of total number of directions $N$ in the extreme cases. Figure 3 exhibits normalized singular values and map of $\mathscr{F}(\mathbf{x})$ with small number of $N=5$ when $\lambda=0.4$. Based on Remark 4 , the value of $N$ must be sufficiently large so, as we expected, locations of $\Sigma_{m}$ cannot be identified via the map of $\mathscr{F}(\mathbf{x})$ with small $N$.

Opposite to the previous result, Figure 4 displays normalized singular values and maps of $\mathscr{F}(\mathbf{x})$ with large number of $N=256$ when $\lambda=0.4$. Similar to the results in Figure 2 , locations of $\Sigma_{m}$ can be examined clearly via the selection of first 3-singular values. Applying 0.1-threshold, it is very hard to identify locations of $\Sigma_{m}$ but, opposite to the result in Figure 2, their locations can be recognized even though some artifacts still exist.

On the basis of recent works [13, 20], it has been confirmed that MUSIC is robust with respect to the random noise. In order to examine the robustness, assume that $10 \mathrm{~dB}$ Gaussian random noise is added to the unperturbed data $u_{\text {far }}\left(\boldsymbol{\vartheta}_{j}, \boldsymbol{\theta}_{l}\right)$. Throughout results in Figure 5 when $N=32$ and $\lambda=0.3$, although some blurring appears in the map of $\mathscr{F}(\mathbf{x})$, we can easily find proper singular values and obtain an accurate image. It is interesting to observe that, opposite to the results in Figure 2, locations of $\Sigma_{m}$ can be detected despite existence of some artifacts.

From the above results, we can examine that, by having small perturbations of random scatterers $\Delta_{s}$, their effects to the scattered fields are quite small so that $\Sigma_{m}$ can be discriminated very accurately. Opposite to the this examination, let us consider the effect of $\Delta_{s}$ when their size and permittivities satisfy $r_{s}=0.1$ and $\varepsilon_{s}=\eta(2.5,3)$, respectively (remember that $r_{m}=0.1$ and $\varepsilon_{m} \equiv 3$ for all $m$ ). In this example, it is very hard to discriminate nonzero singular values associated with $\Sigma_{m}$ so that it is impossible to detect their exact locations; refer to Figure 6 when $N=32$ and $\lambda=0.4$. 


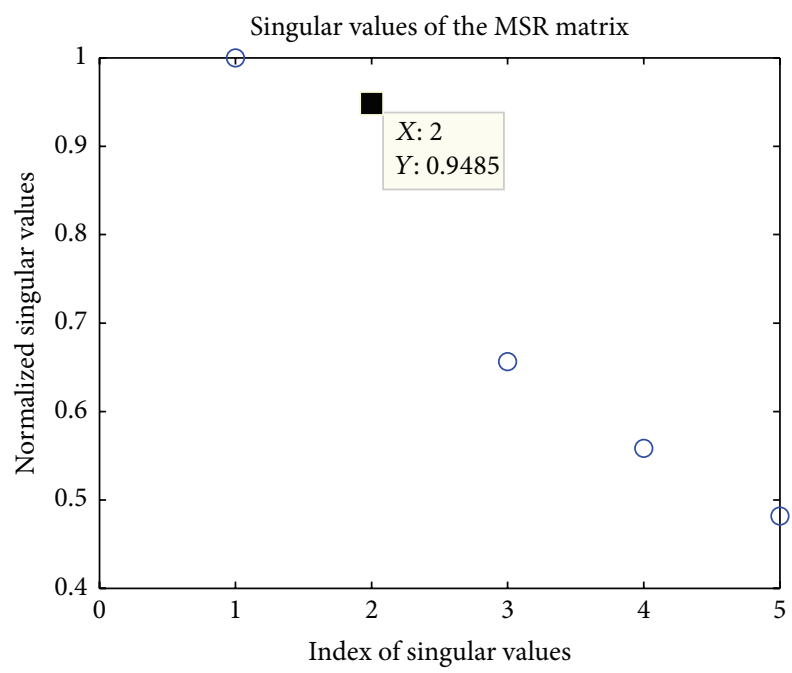

(a)

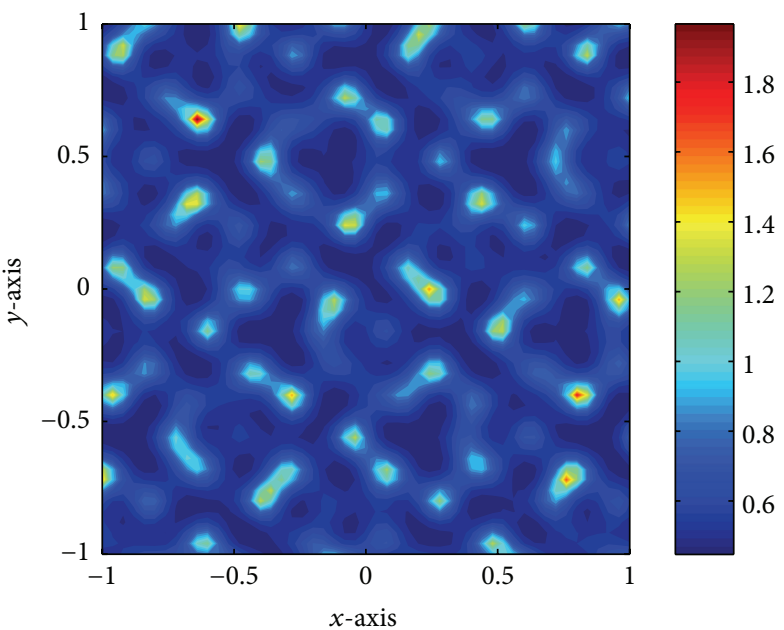

(b)

FIGURE 3: Distribution of normalized singular values (a) and map of $\mathscr{F}(\mathbf{x})$ with first 2-singular values (b).

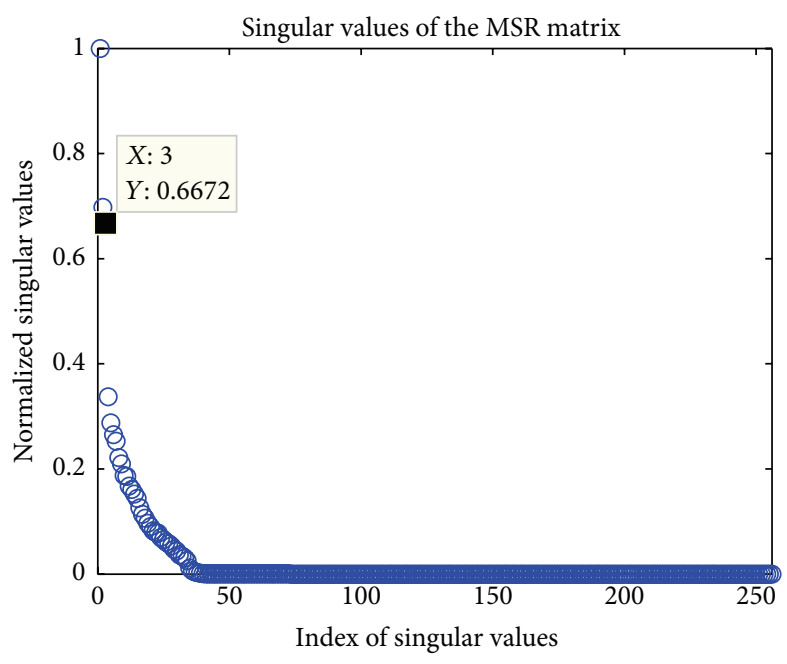

(a)

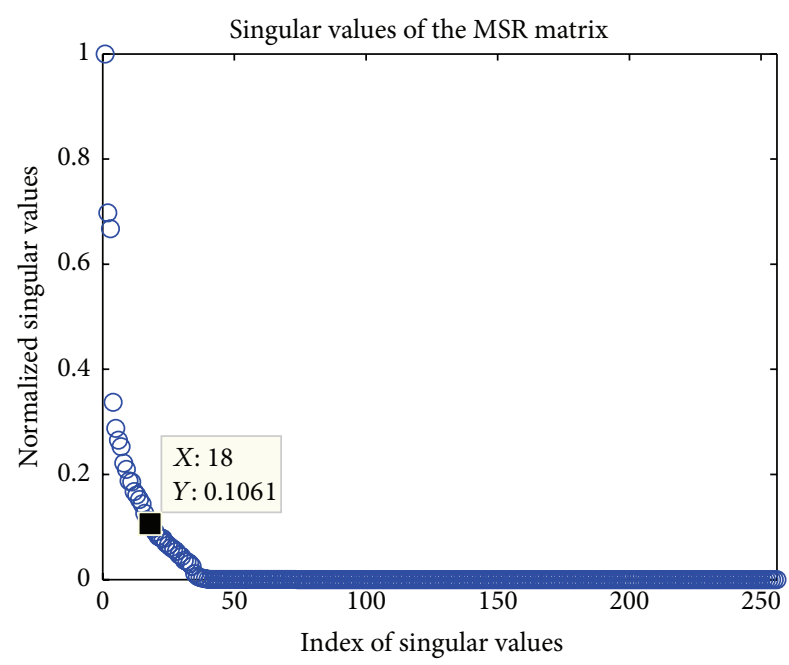

(c)

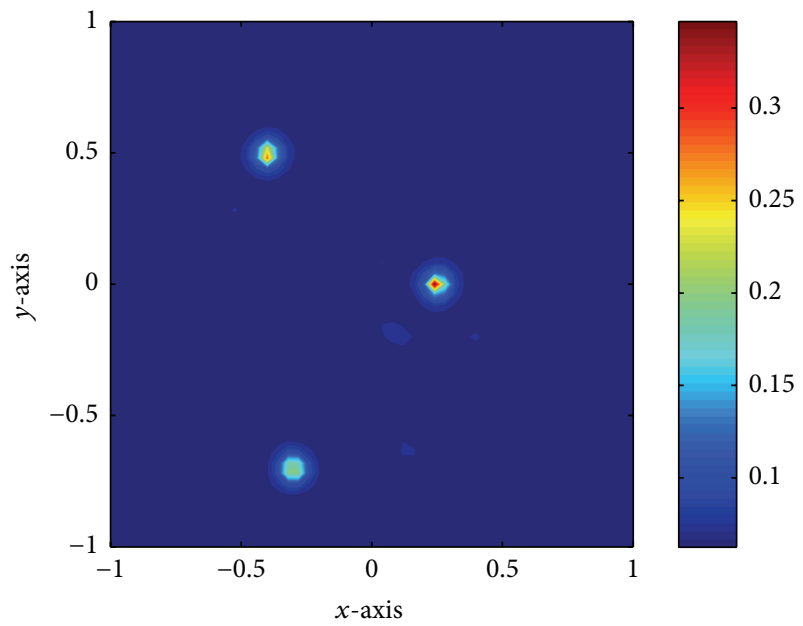

(b)

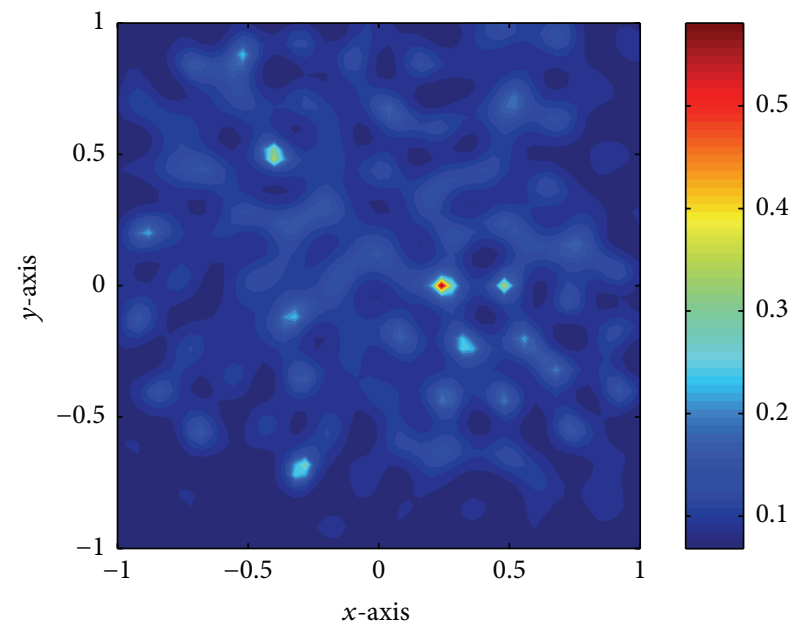

(d)

Figure 4: Distribution of normalized singular values (a, c) and maps of $\mathscr{F}(\mathbf{x})$ with first 3 -singular values (b) and with 0.1 -threshold scheme (d). 


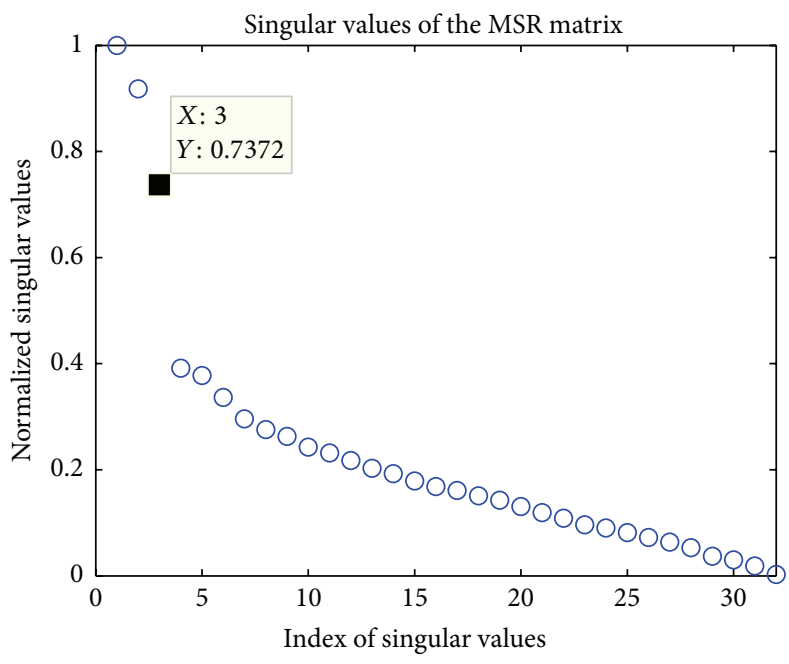

(a)

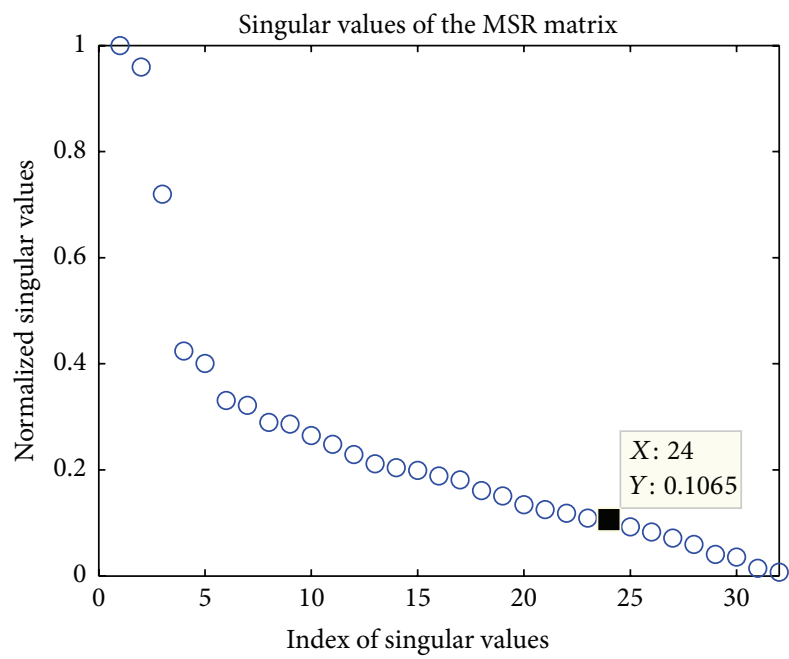

(c)

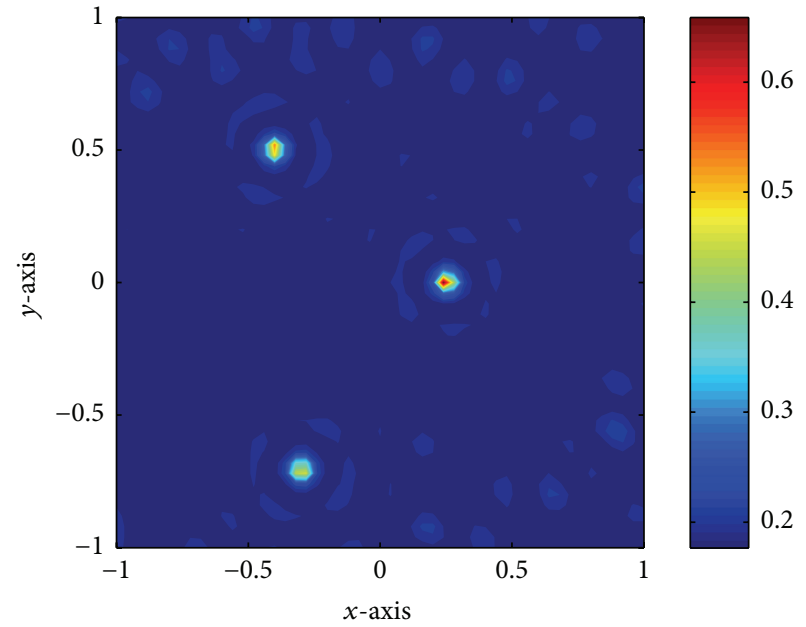

(b)

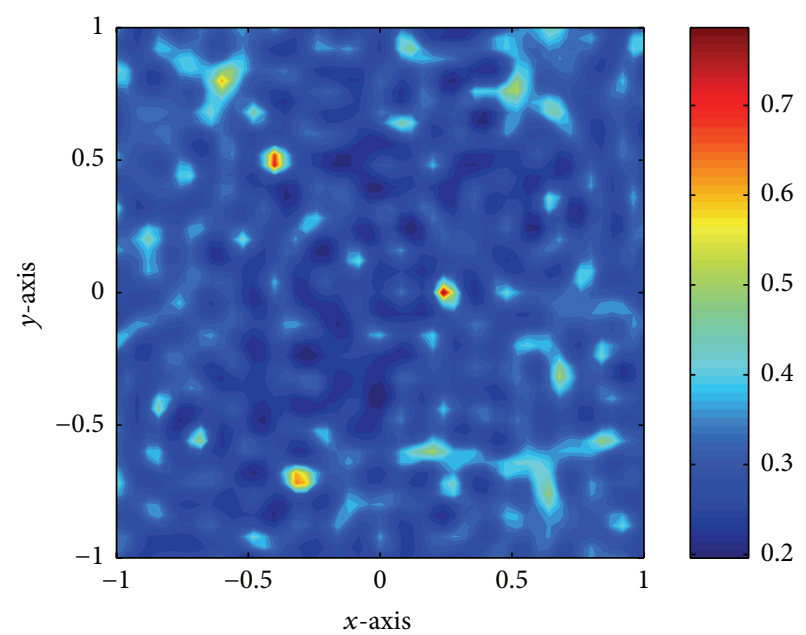

(d)

Figure 5: Distribution of normalized singular values (a, c) and maps of $\mathscr{F}(\mathbf{x})$ with first 3-singular values (b) and with 0.1-threshold scheme (d) when $N=32$ and $\lambda=0.3$, and collected far-field data is perturbed by a white Gaussian random noise.

It is well-known that using multifrequency improves the imaging performance; refer to [13,32-34]. At this moment, we consider multifrequency MUSIC-type imaging in order to compare the imaging performance against the traditional single-frequency one. For given $F$-different frequencies $0<$ $\omega_{1}<\omega_{2}<\cdots<\omega_{F}$, SVD of MSR matrix $\mathbb{K}\left(\omega_{f}\right)$ is

$$
\begin{aligned}
\mathbb{K}\left(\omega_{f}\right) \approx & \sum_{m=1}^{3 M} \sigma_{m}\left(\omega_{f}\right) \mathbf{U}_{m}\left(\omega_{f}\right) \mathbf{V}_{m}^{*}\left(\omega_{f}\right) \\
& +\sum_{s=3 M+1}^{3 M+3 S} \sigma_{s}\left(\omega_{f}\right) \mathbf{U}_{s}\left(\omega_{f}\right) \mathbf{V}_{s}^{*}\left(\omega_{f}\right)
\end{aligned}
$$

Then, by choosing test vector

$$
\mathbf{f}\left(\mathbf{x} ; \omega_{f}\right)=\frac{1}{\sqrt{N}}\left[e^{i \omega_{f} \boldsymbol{\theta}_{1} \cdot \mathbf{x}}, e^{i \omega_{f} \boldsymbol{\theta}_{2} \cdot \mathbf{x}}, \ldots, e^{i \omega_{f} \boldsymbol{\theta}_{N} \cdot \mathbf{x}}\right]^{T}
$$

we can survey the projection operator onto the null (or noise) subspace such that

$$
\begin{aligned}
& \mathbf{P}_{\text {noise }}\left(\mathbf{f}\left(\mathbf{x} ; \omega_{f}\right)\right) \\
& \quad:=\left(\mathbb{q}_{N}-\sum_{m=1}^{3 M+3 S} \mathbf{U}_{m}\left(\omega_{f}\right) \mathbf{U}_{m}^{*}\left(\omega_{f}\right)\right) \mathbf{f}\left(\mathbf{x} ; \omega_{f}\right),
\end{aligned}
$$




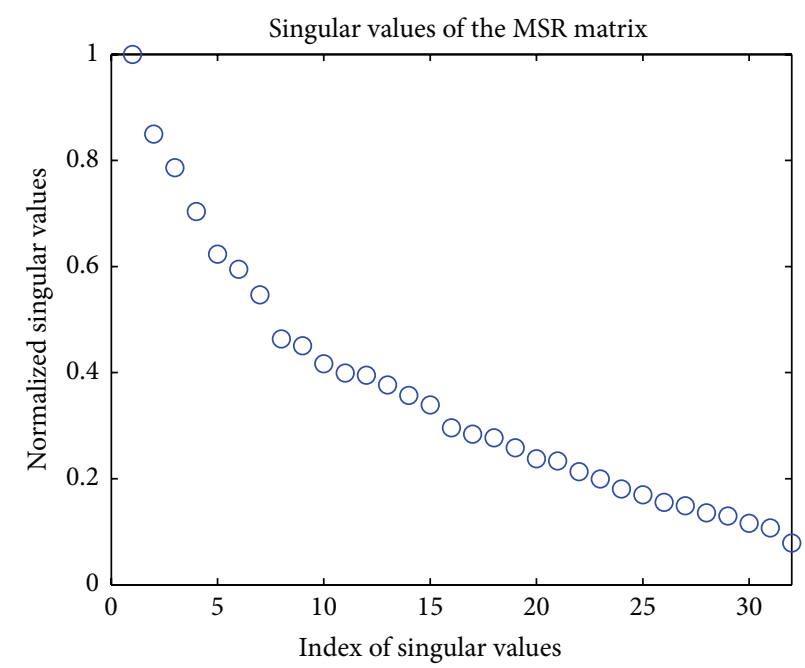

(a)

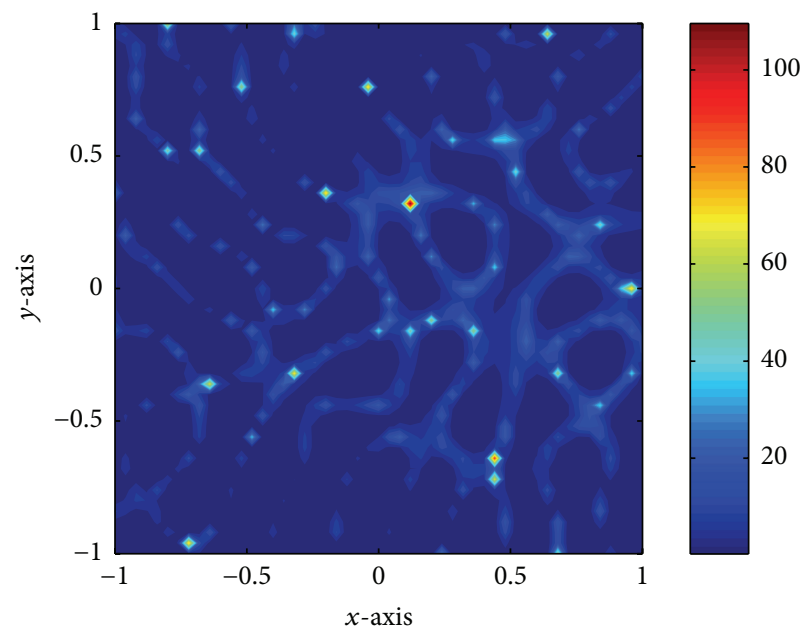

(b)

Figure 6: Distribution of normalized singular values (a) and map of $\mathscr{F}(\mathbf{x})$ with 0.1 -threshold scheme (b).

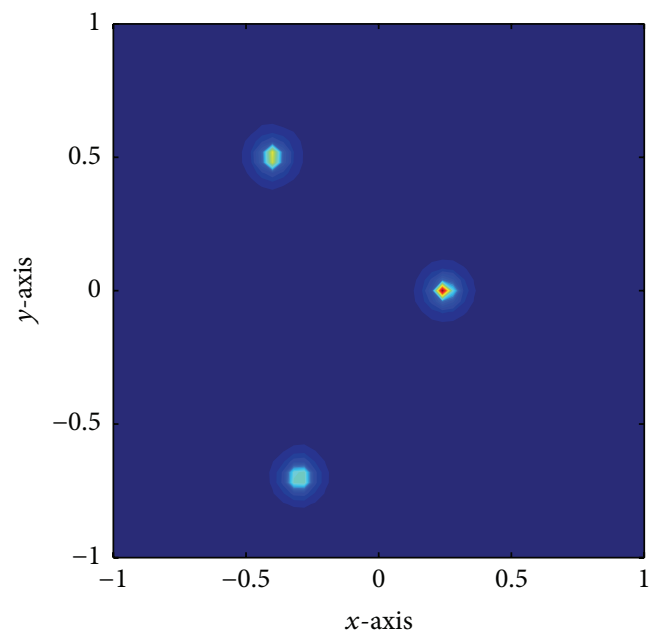

(a)

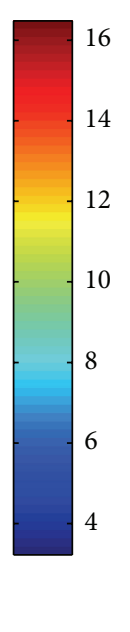

Figure 7: Maps of $Q(\mathbf{x} ; 10)$ with first 3-singular values (a) and with 0.1-threshold scheme (b). and correspondingly multifrequency MUSIC-type imaging function $\mathbb{Q}(\mathbf{x} ; F)$ can be introduced as

$$
\mathbb{Q}(\mathbf{x} ; F)=\left|\frac{1}{F} \sum_{f=1}^{F} \mathbf{P}_{\text {noise }}\left(\mathbf{f}\left(\mathbf{x} ; \omega_{f}\right)\right)\right|^{-1} .
$$

Figure 7 shows maps of $\mathbb{Q}(\mathbf{x} ; 10)$, where $\omega_{f}=2 \pi / \lambda_{s}$. Here, $N=32$ directions are applied and $\lambda_{f}$ are equidistributed in the interval $\left[\lambda_{F}, \lambda_{1}\right]$ with $\lambda_{1}=0.7$ and $\lambda_{F}=$ 0.3. By comparing results in Figure 2, we can observe that unexpected artifacts have been eliminated so that applying

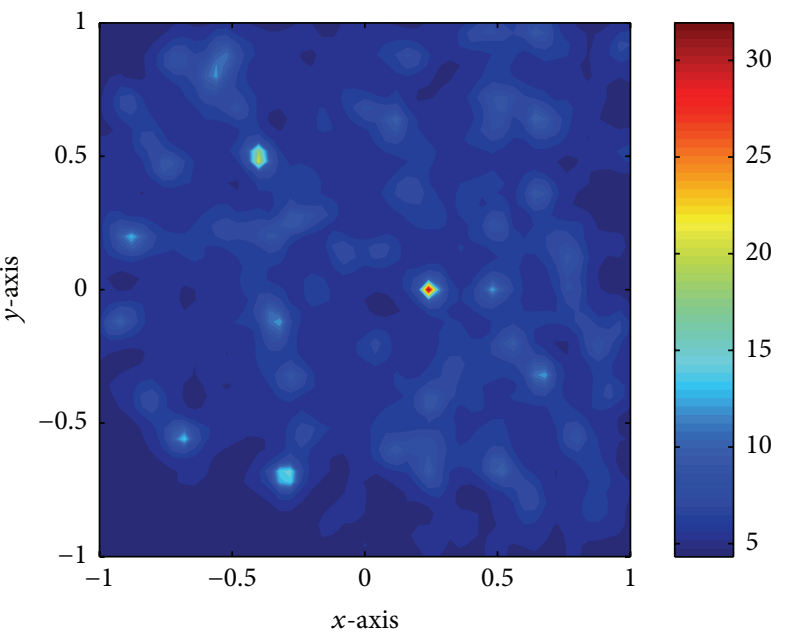

(b) 
Based on recent work [7], the electric field $\mathbf{E}$ in the existence of small inhomogeneity with radius $r$ can be expressed as follows:

$$
\begin{gathered}
\mathbf{E}(\mathbf{x})=\mathbf{E}_{0}(\mathbf{x})+r^{3}\left|\mathbf{B}_{m}\right| \sum_{m=1}^{M}\left(k^{2} \frac{3\left(\varepsilon_{m}-\varepsilon_{0}\right)}{\varepsilon_{m}+2 \varepsilon_{0}} \mathbf{G}\left(\mathbf{x}, \mathbf{z}_{m}\right)\right. \\
\cdot \mathbf{E}_{0}\left(\mathbf{z}_{m}\right)-i \omega \mu_{0} \frac{3\left(\mu_{m}-\mu_{0}\right)}{\mu_{m}+2 \mu_{0}} \nabla \times \mathbf{G}\left(\mathbf{x}, \mathbf{z}_{m}\right) \\
\left.\cdot \mathbf{H}_{0}\left(\mathbf{z}_{m}\right)\right)+O\left(r^{4}\right),
\end{gathered}
$$

where electromagnetic fields $\left(\mathbf{E}_{0}, \mathbf{H}_{0}\right)$ are the solutions of the Maxwell equations

$$
\begin{aligned}
\nabla \times \mathbf{E}_{0} & =i \omega \mu_{0} \mathbf{H}_{0} \quad \text { in } \mathbb{R}^{3}, \\
\nabla \times \mathbf{H}_{0} & =-i \omega \varepsilon_{0} \mathbf{E}_{0}+\mathbf{J}_{0} \\
\lim _{|\mathbf{r}| \rightarrow \infty} \mathbf{r}\left(\nabla \times \mathbf{E}_{0}-i k \frac{\mathbf{r}}{|\mathbf{r}|} \times \mathbf{E}_{0}\right) & =0, \\
\lim _{|\mathbf{r}| \rightarrow \infty} \mathbf{r}\left(\nabla \times \mathbf{H}_{0}-i k \frac{\mathbf{r}}{|\mathbf{r}|} \times \mathbf{H}_{0}\right) & =0
\end{aligned}
$$

and $\mathbf{G}$ is Green's function

$$
\mathbf{G}\left(\mathbf{x}, \mathbf{z}_{m}\right):=\left(\left[\begin{array}{lll}
1 & 0 & 0 \\
0 & 1 & 0 \\
0 & 0 & 1
\end{array}\right]+\frac{\nabla \nabla}{k^{2}}\right) \frac{e^{i k\left|\mathbf{x}-\mathbf{z}_{m}\right|}}{4 \pi\left|\mathbf{x}-\mathbf{z}_{m}\right|} .
$$

Thus, by applying above asymptotic expansion formula and through the similar process in Theorem 3, the result in this paper can be extended to the three-dimensional problem so that MUSIC will be applicable for detecting three-dimensional inhomogeneities surrounded by random scatterers.

In comparison with the MUSIC, other closely related reconstruction algorithms such as linear sampling method [35-37], subspace migration [32, 33, 38], and direct sampling method [39-41] will be applicable for detecting inhomogeneities in random medium. Analysis of imaging functions and exploring their certain properties will be the forthcoming work.

\section{Conflict of Interests}

The author declares that there is no conflict of interests regarding the publication of this paper.

\section{Acknowledgments}

This research was supported by Basic Science Research Program through the National Research Foundation of Korea (NRF) funded by the Ministry of Education (no. NRF2014R1A1A2055225) and the research program of Kookmin University in Korea.

\section{References}

[1] S. R. Arridge, "Optical tomography in medical imaging," Inverse Problems, vol. 15, no. 2, pp. R41-R93, 1999.

[2] H. Ammari, G. Bao, and J. L. Fleming, "An inverse source problem for Maxwell's equations in magnetoencephalography," SIAM Journal on Applied Mathematics, vol. 62, no. 4, pp. 13691382, 2002.

[3] A. S. Fokas, Y. Kurylev, and V. Marinakis, "The unique determination of neuronal currents in the brain via magnetoencephalography," Inverse Problems, vol. 20, no. 4, pp. 1067-1082, 2004.

[4] Y. T. Kim, I. Doh, B. Ahn, and K.-Y. Kim, "Construction of static 3D ultrasonography image by radiation beam tracking method from 1D array probe," Journal of the Korean Society for Nondestructive Testing, vol. 35, no. 2, pp. 128-133, 2015.

[5] S.-H. Son, H.-J. Kim, K.-J. Lee et al., "Experimental measurement system for 3-6 GHz microwave breast tomography," Journal of Electromagnetic Engineering and Science, vol. 15, no. 4, pp. 250-257, 2015.

[6] H. Ammari, E. Iakovleva, and D. Lesselier, "Two numerical methods for recovering small inclusions from the scattering amplitude at a fixed frequency," SIAM Journal on Scientific Computing, vol. 27, no. 1, pp. 130-158, 2005.

[7] H. Ammari, E. Iakovleva, D. Lesselier, and G. Perrusson, "MUSIC-type electromagnetic imaging of a collection of small three-dimensional inclusions," SIAM Journal on Scientific Computing, vol. 29, no. 2, pp. 674-709, 2007.

[8] E. Iakovleva, S. Gdoura, D. Lesselier, and G. Perrusson, "Multistatic response matrix of a 3-D inclusion in half space and MUSIC imaging," IEEE Transactions on Antennas and Propagation, vol. 55, no. 9, pp. 2598-2609, 2007.

[9] Y. Zhong and X. Chen, "MUSIC imaging and electromagnetic inverse scattering of multiple-scattering small anisotropic spheres," IEEE Transactions on Antennas and Propagation, vol. 55, no. 12, pp. 3542-3549, 2007.

[10] H. Ammari, E. Iakovleva, and D. Lesselier, "A MUSIC algorithm for locating small inclusions buried in a half-space from the scattering amplitude at a fixed frequency," Multiscale Modeling \& Simulation, vol. 3, no. 3, pp. 597-628, 2005.

[11] R. Griesmaier, "Reciprocity gap MUSIC imaging for an inverse scattering problem in two layered media," Inverse Problems and Imaging, vol. 3, no. 3, pp. 389-403, 2009.

[12] R. Song, R. Chen, and X. Chen, "Imaging three-dimensional anisotropic scatterers in multilayered medium by multiple signal classification method with enhanced resolution," Journal of the Optical Society of America A, vol. 29, no. 9, pp. 1900-1905, 2012.

[13] H. Ammari, J. Garnier, H. Kang, W.-K. Park, and K. Sølna, "Imaging schemes for perfectly conducting cracks," SIAM Journal on Applied Mathematics, vol. 71, no. 1, pp. 68-91, 2011.

[14] H. Ammari, H. Kang, H. Lee, and W.-K. Park, "Asymptotic imaging of perfectly conducting cracks," SIAM Journal on Scientific Computing, vol. 32, no. 2, pp. 894-922, 2010.

[15] H. Ammari, H. Kang, E. Kim, M. Lim, and K. Louati, "A direct algorithm for ultrasound imaging of internal corrosion," SIAM Journal on Numerical Analysis, vol. 49, no. 3, pp. 1177-1193, 2011.

[16] C. Y. Ahn, K. Jeon, and W.-K. Park, "Analysis of MUSIC-type imaging functional for single, thin electromagnetic inhomogeneity in limited-view inverse scattering problem," Journal of Computational Physics, vol. 291, pp. 198-217, 2015. 
[17] W.-K. Park, "Asymptotic properties of MUSIC-type imaging in two-dimensional inverse scattering from thin electromagnetic inclusions," SIAM Journal on Applied Mathematics, vol. 75, no. 1, pp. 209-228, 2015.

[18] W.-K. Park and D. Lesselier, "MUSIC-type imaging of a thin penetrable inclusion from its multi-static response matrix," Inverse Problems, vol. 25, no. 7, Article ID 075002, 2009.

[19] H. Ammari, J. Garnier, H. Kang, M. Lim, and K. Sølna, "Multistatic imaging of extended targets," SIAM Journal on Imaging Sciences, vol. 5, no. 2, pp. 564-600, 2012.

[20] S. Hou, K. Sølna, and H. Zhao, "A direct imaging algorithm for extended targets," Inverse Problems, vol. 22, no. 4, pp. 1151-1178, 2006.

[21] S. Hou, K. Sølna, and H. Zhao, "A direct imaging method using far-field data," Inverse Problems, vol. 23, no. 4, pp. 1533-1546, 2007.

[22] B. Scholz, "Towards virtual electrical breast biopsy: spacefrequency MUSIC for trans-admittance data," IEEE Transactions on Medical Imaging, vol. 21, no. 6, pp. 588-595, 2002.

[23] H. Ammari and H. Kang, Reconstruction of Small Inhomogeneities from Boundary Measurements, vol. 1846 of Lecture Notes in Mathematics, Springer, Berlin, Germany, 2004.

[24] M. Cheney, "The linear sampling method and the MUSIC algorithm," Inverse Problems, vol. 17, no. 4, pp. 591-595, 2001.

[25] B. Chen, J. J. Stamnes, A. J. Devaney, H. M. Pedersen, and K. Stamnes, "Two-dimensional optical diffraction tomography for objects embedded in a random medium," Pure and Applied Optics: Journal of the European Optical Society Part A, vol. 7, no. 5, pp. 1181-1199, 1998.

[26] L. Borcea, G. Papanicolaou, C. Tsogka, and J. Berryman, "Imaging and time reversal in random media," Inverse Problems, vol. 18, no. 5, pp. 1247-1279, 2002.

[27] A. Kirsch, "The MUSIC algorithm and the factorization method in inverse scattering theory for inhomogeneous media," Inverse Problems, vol. 18, no. 4, pp. 1025-1040, 2002.

[28] B. M. Shevtsov, "Backscattering and inverse problem in random media," Journal of Mathematical Physics, vol. 40, no. 9, pp. 43594373, 1999.

[29] X. Chen, "Multiple signal classification method for detecting point-like scatterers embedded in an inhomogeneous background medium," Journal of the Acoustical Society of America, vol. 127, no. 4, pp. 2392-2397, 2010.

[30] T. Rao and X. Chen, "Analysis of the time-reversal operator for a single cylinder under two-dimensional settings," Journal of Electromagnetic Waves and Applications, vol. 20, no. 15, pp. 2153-2165, 2006.

[31] E. Beretta and E. Francini, "Asymptotic formulas for perturbations of the electromagnetic fields in the presence of thin imperfections," Contemporary Mathematics, vol. 333, pp. 49-63, 2003.

[32] W.-K. Park, "Multi-frequency subspace migration for imaging of perfectly conducting, arc-like cracks in full- and limited-view inverse scattering problems," Journal of Computational Physics, vol. 283, pp. 52-80, 2015.

[33] W.-K. Park, "Analysis of a multi-frequency electromagnetic imaging functional for thin, crack-like electromagnetic inclusions," Applied Numerical Mathematics, vol. 77, pp. 31-42, 2014.

[34] Y.-D. Joh and W.-K. Park, "Structural behavior of the MUSICtype algorithm for imaging perfectly conducting cracks," Progress in Electromagnetics Research, vol. 138, pp. 211-226, 2013.
[35] D. Colton, H. Haddar, and P. Monk, “The linear sampling method for solving the electromagnetic inverse scattering problem," SIAM Journal on Scientific Computing, vol. 24, no. 3, pp. 719-731, 2002.

[36] H. Haddar and P. Monk, "The linear sampling method for solving the electromagnetic inverse medium problem," Inverse Problems, vol. 18, no. 3, pp. 891-906, 2002.

[37] A. Kirsch and S. Ritter, "A linear sampling method for inverse scattering from an open arc," Inverse Problems, vol. 16, no. 1, pp. 89-105, 2000.

[38] Y.-D. Joh and W.-K. Park, "Analysis of multi-frequency subspace migration weighted by natural logarithmic function for fast imaging of two-dimensional thin, arc-like electromagnetic inhomogeneities," Computers \& Mathematics with Applications, vol. 68, no. 12, pp. 1892-1904, 2014.

[39] J. Li, H. Liu, and J. Zou, "Locating multiple multiscale acoustic scatterers," Multiscale Modeling \& Simulation, vol. 12, no. 3, pp. 927-952, 2014.

[40] K. Ito, B. Jin, and J. Zou, "A direct sampling method for inverse electromagnetic medium scattering," Inverse Problems, vol. 29, no. 9, Article ID 095018, 2013.

[41] K. Ito, B. Jin, and J. Zou, "A direct sampling method to an inverse medium scattering problem," Inverse Problems, vol. 28, no. 2, Article ID 025003, 2012. 


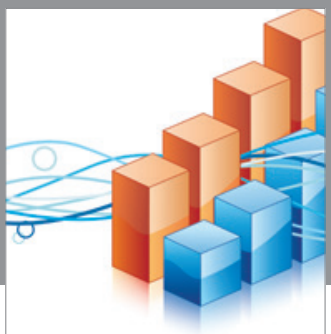

Advances in

Operations Research

vatem alat4

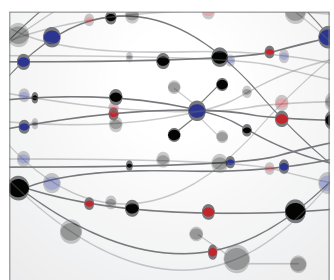

\section{The Scientific} World Journal
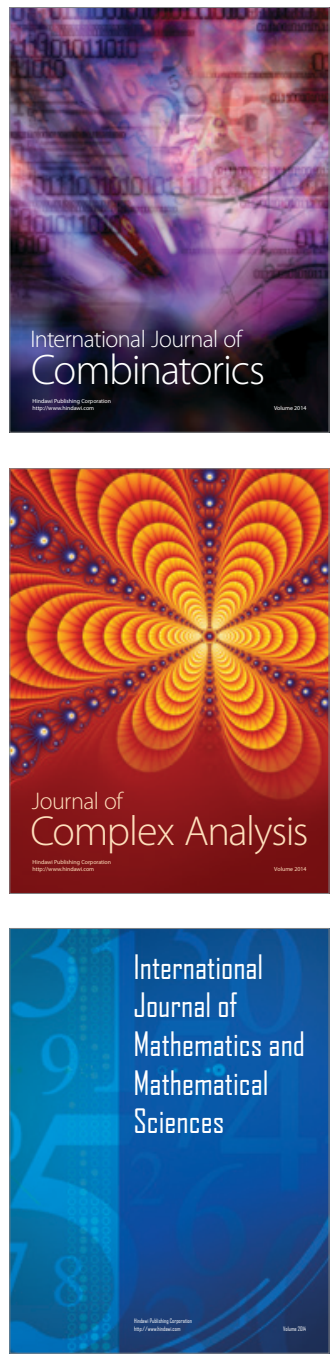
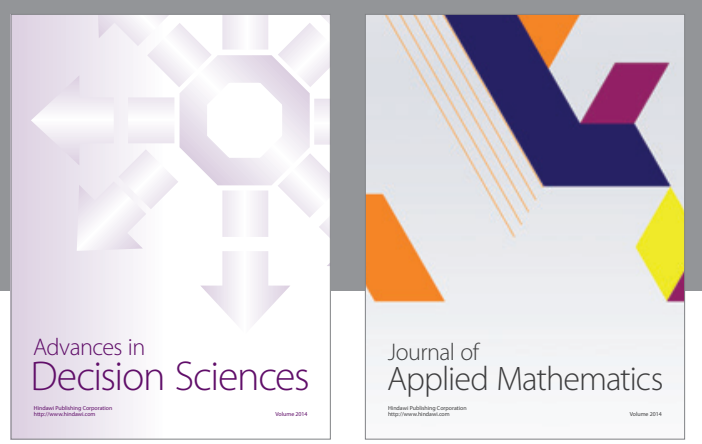

Algebra

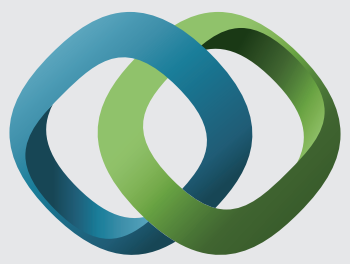

\section{Hindawi}

Submit your manuscripts at

http://www.hindawi.com
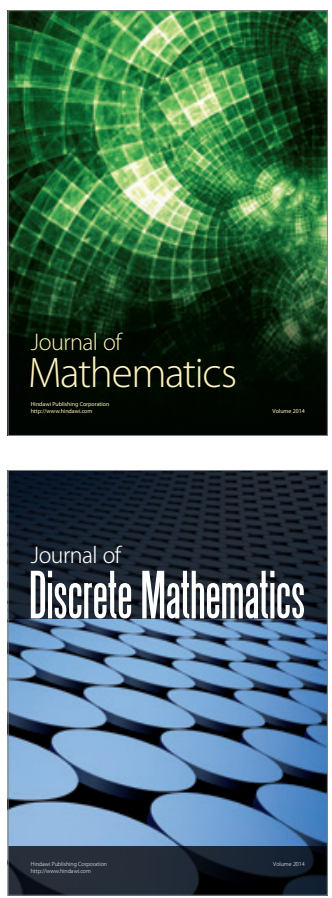

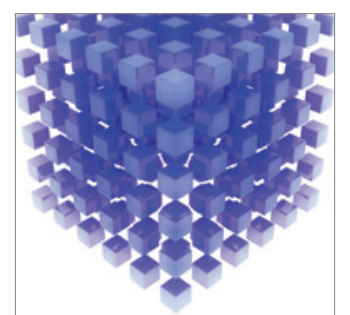

Mathematical Problems in Engineering
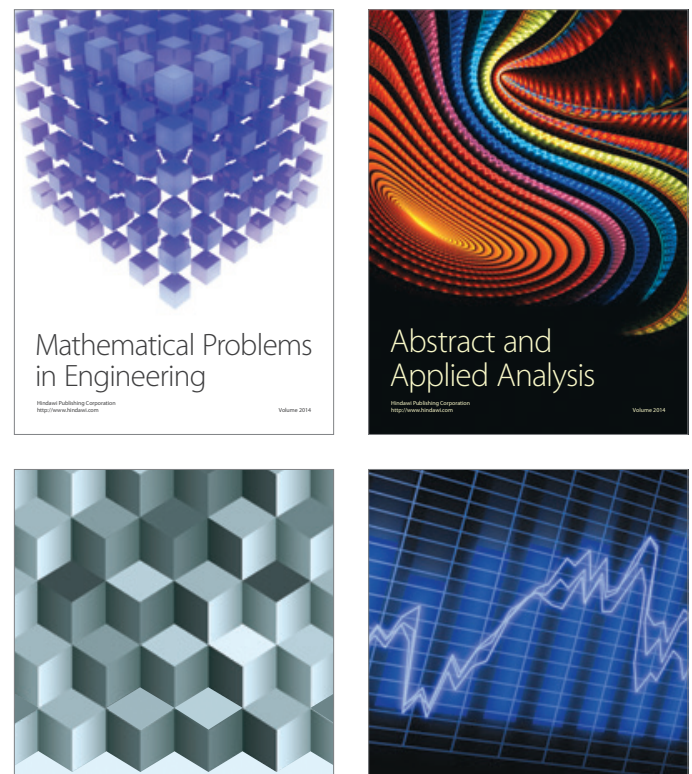

Journal of

Function Spaces

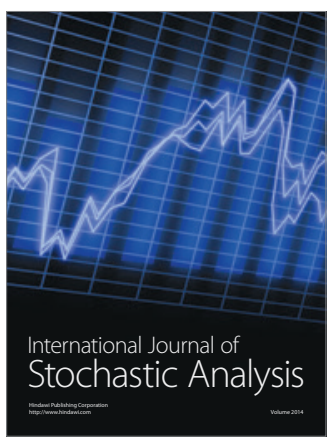

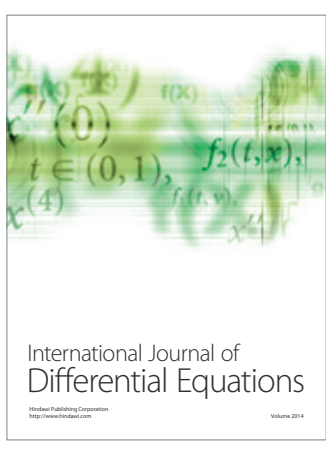
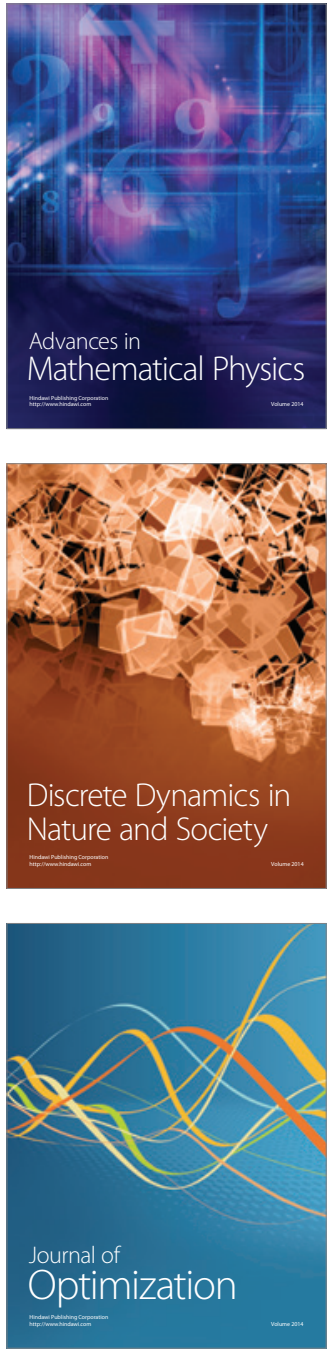